\title{
Mechanical properties of lattice materials via asymptotic homogenization and comparison with alternative homogenization methods
}

\author{
Sajad Arabnejad Khanoki \\ PhD Student, Mechanical Engineering \\ Department, McGill University \\ Montreal, Quebec, Canada, H3A 2K6 \\ Email: \\ sajad.arabnejadkhanoki@mail.mcgill.ca
}

\author{
Damiano Pasini* \\ Associate Professor, Mechanical Engineering \\ Department, McGill University \\ Montreal, Quebec, Canada, H3A 2K6 \\ Email: Damiano.pasini@mcgill.ca
}

\begin{abstract}
Several homogenization schemes exist in literature to characterize the mechanics of cellular materials. Each one has its own assumptions, advantages, and limitations that control the level of accuracy a method can provide. There is often the need in heavy multiscale analyses of lattice materials to find the method that can provide the best trade-off between accuracy and computational cost. In this paper, asymptotic homogenization $(\mathrm{AH})$ is used as a benchmark to test the accuracy of alternative schemes of homogenization applied to lattice materials. AH is first applied to determine the effective elastic moduli and yield strength of six lattice topologies for the whole range of relative density. Yield surfaces are also obtained under multiaxial loading for square, hexagonal, and Kagome lattices, and closed-form expressions of the yield loci are provided for a convenient use in multiscale material problems. With respect to the relative density, the results are then compared to those obtained with other methods available in literature. The analysis shows that the latter can predict the elastic constants with

* Corresponding author: Prof. Damiano Pasini

Phone: + (1)-514-398-6295, Fax: + (1)-514-398-7365

E-mail address: damiano.pasini@mcgill.ca

Mail Address: Room 372, MacDonald Engineering Building, Mechanical Engineering Department, McGill University, Montreal, Quebec, Canada, H3A 2K6
\end{abstract}


an error below $10 \%$ for $\rho<0.25$, whereas for the yield strength the discrepancy is above $20 \%$ for $\rho \geq 0.1$ due to the model assumptions. The results of this work on the effective properties of lattice materials provide not only handy expressions of prompt use in multiscale design problems, but also insight into the level of accuracy that alternative homogenization techniques can attain.

Keywords: lattice materials, periodic cellular materials, multiscale mechanics, asymptotic homogenization, stiffness and strength properties

\section{1- Introduction}

Cellular solids are widely used in applications where weight savings and multifunctional properties are critical. Aerospace sandwich panels, vibration and sound insulators, compact heat exchangers, and biomedical implants are only a few examples of applications. A periodic cellular material can be considered as a microtruss obtained by tessellating a unit cell along independent periodic vectors. Periodic cellular materials with high nodal connectivity have a much higher stiffness and strength per unit mass compared to their counterparts, which have low nodal connectivity with cells arranged in either a regular or disordered pattern, such as in foams [1,2].

In a cellular material, the characteristic length of the unit cell is generally considered several orders of magnitude below the characteristic length of the component. Yet, the study of the unit cell is essential to understand the mechanical response of the material at the macroscale. Several analytical and numerical approaches, as well as experimental investigations, have been proposed in the literature to determine the mechanical properties of cellular materials [3-24]. Their main goal is to obtain the properties of the macromaterial in terms of the effective properties of its unit cell, which is a process of homogenization set to circumvent a detailed - often impractical - analysis of the entire cellular microstructure. Noteworthy contributions in the area of cellular materials are those of Gibson and Ashby [3], Masters 
and Evans [4], Christensen [5], and Wang and McDowell [6, 25], which provide closed-form expressions of the effective mechanical properties. These methods rely on certain premises. They generally assume the cell walls behave like Euler-Bernoulli beams, examine the individual cell wall and determine the elastic constants of the cell by solving deformation and equilibrium problems. They work well for topologies that have a simple arrangement of the cell members, but present limitations if the geometry of the unit cell has a complex topology.

More recently, matrix-based techniques using the Bloch's theorem and the Cauchy-Born hypothesis have been used to homogenize the properties of planar lattice materials [26, 27]. Hutchinson and Fleck [26] first formulated the microscopic nodal deformations of a lattice in terms of the macroscopic strain field, from which the material macroscopic stiffness properties are derived. A methodology was proposed to characterize cell topologies with a certain level of symmetry, e.g. the Kagome lattice and the Triangular-Triangular lattice. Elsayed and Pasini [27] and Elsayed [28] extended this method to deal with planar topologies that can possess any arbitrary geometry of the cell. Vigliotti and Pasini [29, 30] presented a more general matrix-based procedure for the analysis of arbitrary bidimensional and tridimensional cell topologies with open and closed cells.

Other models have been proposed to model the cellular microstructure as an equivalent micropolar medium [8-13]. In micropolar elasticity, in addition to the translational deformation, an independent microscopic rotational field is usually introduced [31, 32]. For a given cell topology, the micropolar elastic constants of the stiffness matrix are obtained through either an explicit structural analysis of the representative unit cell $[11,12]$ or an energy approach [7-10].

Discrete homogenization techniques have been also developed and successfully applied to the characterization of cellular materials [33-37]. With these approaches, the lattice cell walls are modeled with discrete elements such as beam or rod elements. Taylor's expansion of nodal displacement and internal forces are derived and inserted into the equilibrium equations. The discrete sum of equilibrium 
equations is finally converted into a continuous relation of stress and strain from which the homogenized properties are obtained.

Among numerical approaches, asymptotic homogenization (AH) theory has been successfully applied to predict the effective mechanical properties of materials with a periodic microstructure $[14-16,38]$. AH has been widely used not only for the analysis of composite materials and topology optimization of structures [39-42], but also for the characterization of porous materials, such as tissue scaffolds [17, 4345]. AH assumes that any field quantity, such as the displacement, can be described as an asymptotic expansion, which replaced in the governing equations of equilibrium allows to derive the effective properties of the material $[14,16]$. Validation of AH results with experiments has shown that AH is a reliable and accurate method to predict the effective mechanical properties of heterogeneous periodic materials [46-51]. Takano et al. [46] applied AH to analyze micro-macro coupled behaviour of the knitted fabric composite materials under large deformation conditions. The predicted largely deformed microstructures were compared with the experimental results, and a very good agreement was observed. In another study, to validate the accuracy of $\mathrm{AH}$ results, the predicted value of the effective elastic modulus of a porous alumina with $3.1 \%$ porosity was compared with the elastic constant measured from experiments [47], and a relative error of $1 \%$ was found. Guinovart-Díaz et al. [48, 49] computed the thermoelastic effective coefficients of a two-phase fibrous composite using $\mathrm{AH}$. The results were compared with experimental data, and a good agreement was found. In a more recent study, AH has been applied to predict the failure behaviour of three-dimensional weaved composites [51]. The stressstrain response and failure modes of the composite was modeled and shown to match with experimental results. Compared to other homogenization schemes, a noteworthy advantage of $\mathrm{AH}$ is that the stress distribution in the unit cell can be determined accurately and thus be used for a detailed analysis of the strength and damage of the heterogeneous periodic materials [38, 52, 53]. Furthermore, AH has neither limitation on the cell topology nor on the range of relative density; essentially, AH can handle any lattice 
regardless of its relative density. Here for the first time, AH is applied for the characterization of lattice structures. The results are then considered as a benchmark for the comparison with other homogenization methods.

As briefly described above, several methods exist in literature to model the mechanics of cellular materials. Each one has its own assumptions, advantages, and limitations. One central issue to the process of solving a given problem is the careful selection of the homogenization technique that is most effective in terms of accuracy and the most convenient as far the computational cost is concerned. This task, however, presents often trade-offs, which are not always properly defined. For example, closedform expressions, such as those in $[3,6,28,29]$, can be conveniently used to fast compute the effective properties of a lattice material, problems of accuracy might emerge if the microstructure does not respect the model assumptions. The hypothesis that cell walls behave like beam/rod elements ceases to provide reliable results for increasing values of relative density. Moreover, the Euler-Bernoulli beams and rod elements cannot capture the deformation of the solid material at the cell joints, a problem that might affect the estimation of the yield strength of the material. Other techniques, on the other hand, have been proved to be accurate over the whole range of relative densities, but for certain problems they might have the drawback to require longer time of computation [53]. This represents a curb in large multiscale optimization problems of lattice materials [54], because the material properties must be iteratively evaluated several times. In this case, the trade-off solution would be to prefer a method which is computationally faster, as long as it is used in a range of relative density where the results are considered satisfactory within an acceptable range of error. It is thus essential to be able to contrast the validity of alternative homogenization schemes so as to select the most effective method to solve a given problem.

The main goal of this work is to provide a comparative study on the accuracy of classical and more recent homogenization techniques $[3,6,28,29]$ with respect to the governing variable of the lattice, the relative density. Due to its proved superiority, we chose $\mathrm{AH}$ as a benchmark method and review its 
fundamentals in the next section. Six lattice topologies (Figure 1), representative of either bending or stretching dominated behaviour, are selected to test the methods in characterizing their effective elastic constants and mechanical strength. For three common cell topologies, square, hexagonal, and Kagome, AH is used to obtain yield surfaces under multiaxial loading condition. As part of the analysis, approximated closed-form expressions of the results are also given in section 3 for a practical use in multiscale analysis and design problems of cellular materials [55-57]; in particular, they might serve for i) rapid calculation of the effective mechanical properties of lattice materials, ii) validation purposes of experimental data [21, 49], and iii) topology optimization problems, as described in the work of Hassani and Hinton [39], and Liu et. al [58]. In section 4, the comparative analysis on the accuracy of methods is presented; for given ranges of density, the relative error of the properties predicted by each technique is discussed with respect to those obtained with AH.

\section{2- Asymptotic homogenization (AH) method}

A full scale simulation of heterogeneous material with explicit modeling of microstructural features can be very lengthy and time-consuming. Methods of mechanical analysis have therefore sought to obtain the effective mechanical properties of the material by analyzing a representative region of the material, commonly called the representative volume element (RVE). The material is then replaced by an equivalent homogeneous solid with effective properties obtained from the RVE analysis. The distribution of field quantities within the material can then be computed by the analysis of the homogenous structure. This procedure is schematically depicted in Figure 2. A body $\Omega^{\varepsilon}$ with a periodic microstructure subjected to the traction $t$ at the traction boundary $\Gamma_{t}$, a displacement $\mathrm{d}$ at the displacement boundary $\Gamma_{d}$, and a body force $\mathrm{f}$ is replaced by a homogenized body $\Omega$ with prescribed external and traction boundaries applied to $\Omega^{\varepsilon}$, as well as with no geometrical details and voids of the local coordinate system. There are a number of RVE approaches for the analysis of heterogeneous 
materials [59, 60]; here, $\mathrm{AH}$ is applied to determine the effective mechanical properties of the cell topologies under investigation [38]. $\mathrm{AH}$ is a well-developed theory, with a sound mathematical foundation. It has been experimentally tested and proved to provide results that are consistent with those obtained from experiments.

The underlying assumption of $\mathrm{AH}$ is that each field quantity depends on two different scales: one on the macroscopic level $x$, and the other on the microscopic level, $y=x / \varepsilon . \varepsilon$ is a magnification factor that scales the dimensions of the unit cell to the dimensions of the material at the macroscale. It also assumes that field quantities, such as displacement, stress, and strain, vary smoothly at the macroscopic level, and are periodic at the microscale $[14,59]$. Based on $\mathrm{AH}$, each physical field, such as the displacement field, $u$, in a porous elastic body, can be expanded into a power series with respect to $\varepsilon$ :

$$
u^{\varepsilon}(x)=u_{0}(x, y)+\varepsilon u_{1}(x, y)+\varepsilon^{2} u_{2}(x, y)+\cdots
$$

where the functions $u_{0}, u_{1}, u_{2} \ldots$ are Y-periodic with respect to the local coordinate $y$, which means they yield identical values on the opposing sides of the unit cell. $u_{1}$ and $u_{2}$ are perturbations in the displacement field due to the microstructure. $u_{0}$ can be shown to depend only on the macroscopic scale and to be the average value of the displacement field [14]. Taking the derivative of the asymptotic expansion of displacement field with respect to $x$ and using the chain rule allows the small deformation strain tensor to be written as:

$$
\{\varepsilon(u)\}=\frac{1}{2}\left[\left(\nabla u_{0}^{T}+\nabla u_{0}\right)_{x}+\left(\nabla u_{1}^{T}+\nabla u_{1}\right)_{y}\right]+O(\varepsilon)
$$

Where $(\cdot)_{x}$ and $(\cdot)_{y}$ are gradients of the field quantity with respect to the global and local coordinate systems, respectively. Neglecting terms of $O(\varepsilon)$ and higher, the following strain tensors can be defined:

$$
\{\varepsilon(u)\}=\{\bar{\varepsilon}(u)\}+\left\{\varepsilon^{*}(u)\right\},\{\bar{\varepsilon}(u)\}=\frac{1}{2}\left[\left(\nabla u_{0}^{T}+\nabla u_{0}\right)_{x}\right],\left\{\varepsilon^{*}(u)\right\}=\frac{1}{2}\left[\left(\nabla u_{1}^{T}+\nabla u_{1}\right)_{y}\right]
$$


where $\{\bar{\varepsilon}(u)\}$ is the average or macroscopic strain, and $\left\{\varepsilon^{*}(u)\right\}$ is the fluctuating strain varying periodically at the microscale level. Substituting the strain tensor into the standard weak form of the equilibrium equations for a cellular body $\Omega^{\varepsilon}$ with the pertinent geometrical details of voids and cell wall, yields the following equation [59]:

$$
\int_{\Omega^{\varepsilon}}\left\{\varepsilon^{0}(v)+\varepsilon^{1}(v)\right\}^{T}[\mathrm{E}]\left\{\bar{\varepsilon}(u)+\varepsilon^{*}(u)\right\} d \Omega^{\varepsilon}=\int_{\Gamma_{t}}\{\mathrm{t}\}^{T}\{\mathrm{v}\} d \Gamma
$$

where $[E]$ is the local elasticity tensor that depends on the position within the RVE, $\left\{\varepsilon^{0}(v)\right\}$ and $\left\{\varepsilon^{1}(v)\right\}$ are the virtual macroscopic and microscopic strains, respectively, and $\{\mathbf{t}\}$ is the traction at the traction boundary $\Gamma_{t}$. Being the virtual displacement, $\{\mathbf{v}\}$ may be chosen to vary only on the microscopic level and be constant on the macroscopic level. Based on this assumption, the microscopic equilibrium equation can be obtained as:

$$
\int_{\Omega^{\varepsilon}}\left\{\varepsilon^{1}(v)\right\}^{T}[\mathrm{E}]\left\{\bar{\varepsilon}(u)+\varepsilon^{*}(u)\right\} d \Omega^{\varepsilon}=0
$$

Taking the integral over the RVE volume ( $\left.V_{R V E}\right)$, equation (5) may be rewritten as:

$$
\int_{V_{R V E}}\left\{\varepsilon^{1}(v)\right\}^{T}[\mathrm{E}]\left\{\varepsilon^{*}(u)\right\} d V_{R V E}=-\int_{V_{R V E}}\left\{\varepsilon^{1}(v)\right\}^{T}[\mathrm{E}]\{\bar{\varepsilon}(u)\} d V_{R V E}
$$

The above equation represents a local problem defined on the RVE. For a given applied macroscopic strain, the material can be characterized if the fluctuating strain, $\left\{\varepsilon^{*}(u)\right\}$, is known. The periodicity of the strain field is ensured by imposing periodic boundary conditions on the RVE edges (Figure 3); the nodal displacements on the opposite edges are set to be equal $[59,61]$.

Equation (6) can be discretized and solved via finite element analysis as described in [15, 16, 52, 59]. For this purpose, equation (6) can be simplified to obtain a relation between the microscopic displacement field $\{\mathrm{D}\}$ and the force vector $\{\mathrm{f}\}$ as: 


$$
[\mathrm{K}]\{\mathrm{D}\}=\{\mathrm{f}\}
$$

where $[\mathrm{K}]$ is the global stiffness matrix defined as:

$$
[\mathrm{K}]=\sum_{e=1}^{m}\left[\mathrm{k}^{e}\right],\left[\mathrm{k}^{e}\right]=\int_{Y^{e}}[\mathrm{~B}]^{T}[\mathrm{E}][\mathrm{B}] d Y^{e}
$$

with $\sum_{e=1}^{m}(\cdot)$ the finite element assembly operator, $m$ the number of elements, $[\mathrm{B}]$ the strain-displacement matrix, and $Y^{e}$ the element volume. The force vector $\{f\}$ in equation (7) is expressed as:

$$
\{\mathrm{f}\}=\sum_{e=1}^{m}\left\{\mathrm{f}^{e}\right\},\left\{\mathrm{f}^{e}\right\}=\int_{Y^{e}}[\mathrm{~B}][\mathrm{E}]\{\bar{\varepsilon}(u)\} d Y^{e}
$$

Equation (9) can be either used for the linear elastic analysis of the microstructure or to model the effect of material nonlinearity as a result of elastoplasticity deformation of the unit cell. The material yield strength and the effective elastic modulus can be characterized by the linear analysis of the microstructure, while the ultimate strength of the material can be obtained through elastoplasticity analysis. A detailed discussion on elastoplasticity analysis of lattice microstructures using $\mathrm{AH}$ is provided in a previous study [62].

Considering the assumption of small deformation and elastic material behavior, the solution of equation (7) leads to a linear relation between the macroscopic $\{\bar{\varepsilon}(u)\}$ and microscopic $\{\varepsilon(u)\}$ strain through the local structural tensor $[\mathrm{M}]$ :

$$
\{\varepsilon(u)\}=[\mathrm{M}]\{\bar{\varepsilon}(u)\}
$$

For a two-dimensional case, three independent unit strains are required to construct the [M] matrix.

$$
\bar{\varepsilon}_{11}=\left[\begin{array}{lll}
1 & 0 & 0
\end{array}\right]^{T}, \bar{\varepsilon}_{22}=\left[\begin{array}{lll}
0 & 1 & 0
\end{array}\right]^{T}, \bar{\varepsilon}_{12}=\left[\begin{array}{lll}
0 & 0 & 1
\end{array}\right]^{T}
$$

The macroscopic strains are applied to equation (9) to obtain the force vector for the computation of microscopic displacements through equation (7). Using the strain-displacement matrix [B], the 
through equation (3). The local structural tensor $[\mathrm{M}]$ can then be obtained at the element centroid by solving three sets of matrix equations (for 2D) once $\{\bar{\varepsilon}(u)\}$ and $\{\varepsilon(u)\}$ are known. Here, since three independent unit strains are considered, each column of the matrix $[\mathrm{M}]$ represents the microscopic strain tensor $\{\varepsilon(u)\}$. The effective stiffness matrix can be simply derived by taking the integral of the microscopic stress over the RVE and dividing by the RVE volume:

$$
\{\bar{\sigma}\}=\frac{1}{\left|V_{R V E}\right|} \int_{V_{R V E}}[\mathrm{E}][\mathrm{M}] d V_{R V E} \bar{\varepsilon}
$$

from which the effective stiffness matrix $\left[\mathrm{E}^{\mathrm{H}}\right]$ can be defined as

$$
\left[\mathrm{E}^{\mathrm{H}}\right]=\frac{1}{\left|V_{R V E}\right|} \int_{V_{R V E}}[\mathrm{E}][\mathrm{M}] d V_{R V E}
$$

The homogenized stiffness matrix relates the macroscopic strains to the macroscopic stresses of the homogenized material. AH also allows obtaining the macroscopic stresses that lead to the microscopic yield, or the endurance limit, as well as fracture. To calculate the yield strength of the unit cells, the microscopic stress distribution $\{\sigma\}$ corresponding to the multiaxial macroscopic stress $\{\bar{\sigma}\}$ can be obtained via the following equation:

$$
\{\sigma\}=[\mathrm{E}][\mathrm{M}]\left[\mathrm{E}^{\mathrm{H}}\right]^{-1}\{\bar{\sigma}\}
$$

The von Mises stress distribution at the microstructure can then be used to capture the yield surface of the unit cell expressed as:

$$
\left\{\bar{\sigma}^{y}\right\}=\frac{\sigma_{y s}}{\max \left\{\sigma_{\mathrm{vM}}(\bar{\sigma})\right\}}\{\bar{\sigma}\}
$$


where $\left\{\bar{\sigma}^{y}\right\}$ is the yield surface of the unit cell, $\sigma_{y s}$ is the yield strength of the bulk material, and $\sigma_{\mathrm{vM}}(\cdot)$ is the von Mises stress at the microstructure corresponding to the applied macroscopic stress. To calculate the yield strength of the unit cells under uni-axial tension in $\mathrm{x}$ and $\mathrm{y}$ directions, and pure shear, macroscopic unit stresses can be applied to equation (15). $\bar{\sigma}_{x x}^{y}, \bar{\sigma}_{y y}^{y}$, and $\bar{\tau}_{x y}^{y}$ can be denoted, respectively, as the macroscopic yield strength of the unit cell under uniaxial tension in $\mathrm{x}$, and $\mathrm{y}$ direction, and under pure shear. To generate the yield surface of the unit cell under multiaxial loadings, the procedure summarized above is repeated for alternative combinations of multiaxial stresses.

It should be noted that the results presented here are based on the analysis of RVE under periodic boundary conditions. Therefore, it is assumed the RVE is far from the domain boundary, where local effects, including local stress heterogeneities, and size and edge effects, do not occur. The effective mechanical properties obtained here can, therefore, be used to capture the average of macroscopic field quantities over the lattice components, and not local stress heterogeneities. To capture these local effects, AH can be integrated with the finite element mesh superposition method [63-65] or an adaptive multiscale methodology [66].

\section{3- Effective mechanical properties of cellular structures by AH}

For the representative lattices in Figure 1, we apply AH to obtain the effective stiffness and yield strength as a function of the relative density. To capture the effective characteristic of each cell topology, regardless of the solid material, the properties are normalized with respect to the base material. A parametric geometry of the unit cell is created assuming cell walls with uniform thickness, which - in turn - is varied at discrete values to span the whole range of relative density. ANSYS (Canonsburg, Pennsylvania, U.S.A) is used to build, mesh, and solve the 2D problem of the lattice material, which is modelled with planar eight-node elements (Plane 82). 
Figure 4 illustrates the homogenized elastic constants of the cell topologies as a function of relative density. As can be seen, the effective Young's modulus, shear modulus, and Poisson's ratios converge to the elastic constants of the base solid material as the relative density reaches one. Figure 4 shows that Young's modulus of hexagonal, triangular, and Kagome lattice are equal in both $\mathrm{x}$ and $\mathrm{y}$ direction. These materials have in-plane elastic isotropy due to three-fold symmetry of their lattice microstructure $[1,12,26,28,67]$. On the other hand, Square, mixed triangle A and B, are orthotropic, and their Young's modulus are only equal in $\mathrm{x}$ and $\mathrm{y}$ direction. More detail about the connectivity, symmetries, and anisotropy of equivalent continua of considered lattice topologies can be found in literature $[1,12$, $26,28,67]$.

As can be also seen in Figure 4, the square cell has a superior elastic stiffness due to the alignment of the cell walls in the loading direction, but it exhibits very low stiffness under shear loading as a result of cell wall bending. While the hexagon has also low Young's and shear moduli, its Poisson's ratio is high, as shown in Figure $2 \mathrm{~b}$. Mixed triangular A can be obtained by $45^{\circ}$ rotation of its B variant. Due to the anisotropic property of the cell, its effective mechanical properties vary by the rotation of the unit cell. A comparison of effective mechanical properties for Mixed A and B in Figure 4 shows that the elastic modulus of Mixed A is about 30\% lower than Mixed B. On the other hand, shear modulus of Mixed A is about 30\% higher than Mixed B.

Figure 5 shows the yield strength as a function of relative density. The results were obtained by applying three loading conditions (uniaxial load in $\mathrm{x}$ and $\mathrm{y}$ directions, and shear load) to determine the maximum von Mises stress in the cell walls and the initial yield strength of each lattice. For the relative density equal to one, the material is fully dense with yield strength equal to that of its solid material. A common feature in the plots of Figure 5 is a sudden decrease of the effective yield strength for decreasing values of relative density. The reason is the presence of stress concentration at the cell joints, which locally increases the level of stress. As illustrated in Figure 5, the material yield strength 
decreases of about $60 \%$ at relative density of $99 \%$. For the hexagonal, Kagome, and triangular lattice, the yield strength is dissimilar in the $\mathrm{x}$ and $\mathrm{y}$ direction, unlike the other in-plane elastic properties. The anisotropic behaviour of these cell topologies have been also shown in the literature by modeling cell walls with beam/rod elements $[6,25,29]$. The anisotropic behaviour of these lattices will be discussed in more detail in the next section.

While the initial yield strength provided in Figure 5 is limited to the uniaxial and shear forces, many applications require the material to withstand multiaxial loadings. We thus obtain here the yield surfaces for alternative combinations of macroscopic stress. As described in the previous section, the corresponding macroscopic strain is computed and applied to the unit cells. Being in linear elasticity, the location of the yield point on the yield surface of each lattice is obtained by multiplying the macroscopic stress with the ratio of the material yield strength and the maximum von Mises stress. Figure 6-8 show the yield surfaces normalized with respect to the initial yield strength in the uniaxial and shear directions for the hexagonal, square, and Kagome lattices at given relative density. These plots and those in Figures 4 and 5, assist the selection of a cell topology for a given problem. In particular, Figures 6 and 7 refer to the hexagonal and square lattice for the relative density of 50\%, and Figure 8 pertains to the Kagome cell for the relative density of $30 \%$. We selected $30 \%$ for the Kagome, because for a 0.5 relative density the base material fills almost completely the triangular voids, and thus the Kagome structure cannot be realized.

As can be seen in Figures 6-8, the yield surfaces are controlled by the shape of the unit cell. For example, the yield surface of the hexagonal cell (Figure 6a) resembles a parallelogram. For design purposes, it is often convenient to resort to closed-form expressions that can approximately describe the geometry of a yield surface. For the hexagonal, square, and Kagome unit cells, Table 1 lists the functions with relative fitting parameters of their yield surfaces. For the hexagonal cell (Figure 6a), $m_{1}$ and $m_{2}$ are the slopes of the parallelogram lines expressed as a function of relative density. For the 
Kagome cell, the approximated surfaces is also parallelogram, while for the square cell, a pyramid with an elliptical base has been used to resemble the yield surface.

We note here that the results illustrated in Figures 4-8 and the expressions given in Table 1 can be conveniently used in multiscale design and topology optimization problems of cellular materials $[39,55-$ 58], where the effective mechanical properties are required as a function of relative density. They can also be used to generate an efficient data base for the validation of experimental results, as recently suggested in [21, 49].

\section{4- Comparative analysis of homogenization schemes and discussion}

Although AH has been proved to have no inherent limitation on the cell topology and to provide consistent results for the whole range of relative density, its main drawback is often considered to be the computational cost. This can be high if the problem at hand is complex and of multiscale nature, as well as if it contains a large number of variables [53]. In such instances, less precise but faster methods might be preferred. This section discusses the validity and compares the accuracy of classical and more recent homogenization techniques $[3,6,28,29]$, with respect to $\mathrm{AH}$, chosen here as a benchmark.

The closed-form expressions of the effective elastic modulus and yield strength obtained via alternative methods $[3,6,28,29]$ are given in Appendix and plotted in Figure 9 as a function of relative density. For the cell topologies shown in Figure 1, the properties are contrasted for relative density lower than $0.3(\rho<0.3)$, where the assumption of Euler-Bernoulli beams considered by the selected methods holds. It should be noted that for the range of relative density lower than 0.3 only the linear part of relative density equation is considered, as the quadratic part does not have a significant influence on the relative density of the cell. This is consistent with results previously obtained [3, 6, 28, 29].

Figure 9 shows that by decreasing the relative density, the effective mechanical properties converge to the values obtained with $\mathrm{AH}$. The analytic expressions generally underestimate the effective elastic 
constant, with the exception of the Poisson's ratio of the hexagonal cell obtained by Gibson and Ashby [3]. We note that in the study by Wang and McDowell [6], the cell walls of stretching dominated cell were modelled as rod elements, while Elsayed [28] and Vigliotti and Pasini [29] considered cell wall as beam elements. As can be seen, whereas for stretching dominated lattices the contribution of bending moments on the effective elastic constants is almost negligible, this is not the case for bending dominated cells, such as the hexagon. Figure $9 \mathrm{~b}$ shows that if the effect of axial deformation is modelled in the formulation, as in [29], lower values of the effective Young's and shear modulus can be observed.

Figure 10 shows the relative error of each effective property normalized with its respective one obtained via AH. As can be seen, the assumption of modelling cell walls as beams or rod elements yield error less than $10 \%$ for stretching dominated lattices. In contrast, for bending-dominated lattices the error can be much higher. For example, for the square cell the error of the effective shear modulus calculated by Wang and McDowell [6] at $\rho=0.15$ can be as high as $24 \%$.

Figure 11 illustrates the initial yield strength of the cell topologies under uni-axial and pure shear stresses. The results obtained by asymptotic homogenization are also compared to the expressions listed in the Appendix $[3,6,29]$. As can be seen, the analytic expressions overestimate the AH results for high values of relative density, and the deviation increases with increasing relative density. On the other hand, for low range of relative density the results of the analytic expression converge to those of AH. Figure 11 also shows the anisotropic property of hexagonal, Kagome, and triangular lattice. As can be seen in Figure 11b for the hexagonal lattice, if cell walls are modeled with beam elements by considering bending moment as the only deformation mode in the element formulation, the lattice shows isotropic behaviour in terms of mechanical strength [3]. However, anisotropic behaviour can be observed if axial deformation mode is also added to the deformation mode of the beam element formulation [29]. The value of axial forces in the cell walls varies with loading direction resulting in the anisotropic property of hexagonal cell. For Kagome and triangular lattices, both bending moments and 
axial forces change by loading direction, thereby causing the anisotropy behaviour in the mechanical strength of the material. With AH theory, all deformation modes, including bending, axial, and significant shear deformation at cell edges and vertices can be captured. As a result, changes of stress distribution throughout the lattice with the loading direction can be captured with high accuracy, and anisotropic behaviour can be observed.

Similarly to Figure 10, Figure 12 shows the relative errors between the initial yield strengths. In contrast to the range of error in Figure 10 and 12, we observe here that the assumption of beam/rod elements for the cell walls is more accurate for the effective elastic constants. For example, the effective elastic constants of stretching dominated can be estimated with an error less that $10 \%$ for $\rho<0.25$. For the yield strength, on the other hand, the analytic expressions are overpredictive, since the assumption of rod element for the cell walls cannot capture any stress localization. Rod elements can only describe uniaxial deformation mode, and significant deformation cannot be captured at the cell edges and vertices as a result of bending or shear deformation. The accuracy can be improved if model refinements are implemented. For example for Kagome and triangle cells (Figure 12(c, d)), the error considerably reduces if the cell walls are modeled with beam elements, as opposed to rods. For instance at $30 \%$ relative density, an error decrease of over $20 \%$ can be obtained. Furthermore, for the hexagonal cell (Figure 12(b)) the results of the yield strength at low relative density show that the analytic expressions can be more accurate if axial forces, besides bending moments, are modeled in the cell walls. Yet for higher relative density, the relative error rises since the deformation of the solid material at the cell joints cannot be captured. In particular for the hexagon, the relative error of the initial yield strength is above $20 \%$ for $\rho \geq 0.1$. This observation is critical and cannot be neglected if the lattice material is to be designed to withstand cyclic loading. On the other hand with AH, the lattice cell walls are modeled with continuum elements with sufficient mesh density; therefore, all deformation modes including significant deformations in the cell edges and vertices could be captured. 


\section{5- Conclusion}

In the first part of the paper, asymptotic homogenization theory has been applied to a lattice with six cell topologies of either stretching or bending dominated behaviour. The effective elastic modulus and yield strength have been obtained for the whole range of relative density. The yield surfaces for multiaxial loading have been provided for three common cell topologies, i.e. square, hexagonal, and Kagome, and closed-form expressions approximating the geometry of the yield surfaces are given for handy use in applications involving multiscale analyses and design problems of cellular materials.

In the second part, the relative density is used as criterion to compare AH results with those obtained with homogenization schemes available in literature. For stiffness and strength properties, the relative error of each method with respect to $\mathrm{AH}$ is also contrasted. The results have shown that the analytic expressions cannot capture material deformation and stress localization at the cell joints; thus they significantly overpredict the yield strength of a lattice material. For instance, the effective elastic constants of stretching dominated lattices can be estimated with an error below $10 \%$ for $\rho<0.25$, whereas the initial yield strength might yield relative errors above $20 \%$ for $\rho \geq 0.1$. These and other results presented in this work can contribute to gain insight into the level of accuracy that a 
homogenization scheme might offer, and help to select the most accurate and cost-effective technique for a given problem.

\section{6- Appendix}

The table below summarizes the expressions of the effective mechanical properties obtained in literature for various cell topologies.

\section{Reference}

[1] V. Deshpande, M. Ashby, N. Fleck, Foam topology: bending versus stretching dominated architectures, Acta Materialia, 49 (2001) 1035-1040.

[2] M. Ashby, The properties of foams and lattices, Philosophical Transactions A, 364 (2006) 15.

[3] L.J. Gibson, M.F. Ashby, Cellular solids: structure and properties, Cambridge University Press, Cambridge, UK, 1999.

[4] I. Masters, K. Evans, Models for the elastic deformation of honeycombs., Composite structures, 35 (1996) 403-422.

[5] R.M. Christensen, Mechanics of cellular and other low-density materials, International Journal of Solids and Structures, 37 (2000) 93-104.

[6] A. Wang, D. McDowell, In-plane stiffness and yield strength of periodic metal honeycombs, Journal of Engineering Materials and Technology, 126 (2004) 137-156.

[7] A. Askar, A. Cakmak, A structural model of a micropolar continuum, International Journal of Engineering Science, 6 (1968) 583-589.

[8] J. Chen, M. Huang, Fracture analysis of cellular materials: a strain gradient model, Journal of the Mechanics and Physics of Solids, 46 (1998) 789-828.

[9] Z. Bazant, M. Christensen, Analogy between micropolar continuum and grid frameworks under initial stress, International Journal of Solids and Structures, 8 (1972) 327-346.

[10] R. Kumar, D. McDowell, Generalized continuum modeling of 2-D periodic cellular solids, International Journal of Solids and Structures, 41 (2004) 7399-7422.

[11] X. Wang, W. Stronge, Micropolar theory for two-dimensional stresses in elastic honeycomb, Proceedings: Mathematical, Physical and Engineering Sciences, (1999) 2091-2116.

[12] W. Warren, E. Byskov, Three-fold symmetry restrictions on two-dimensional micropolar materials, European Journal of Mechanics-A/Solids, 21 (2002) 779-792.

[13] K. Sab, F. Pradel, Homogenisation of periodic Cosserat media, International Journal of Computer Applications in Technology, 34 (2009) 60-71.

[14] B. Hassani, E. Hinton, A review of homogenization and topology optimization I--homogenization theory for media with periodic structure, Computers \& Structures, 69 (1998) 707-717.

[15] B. Hassani, E. Hinton, A review of homogenization and topology optimization II--analytical and numerical solution of homogenization equations, Computers \& Structures, 69 (1998) 719-738.

[16] J. Guedes, N. Kikuchi, Preprocessing and postprocessing for materials based on the homogenization method with adaptive finite element methods, Computer methods in applied mechanics and engineering, 83 (1990) 143198.

[17] Z. Fang, B. Starly, W. Sun, Computer-aided characterization for effective mechanical properties of porous tissue scaffolds, Computer-Aided Design, 37 (2005) 65-72. 

effective mechanical properties for electromagnetic composite conductor, Journal of Composite Materials, 38 (2004) 1371.

[19] W.-X. Wang, D. Luo, Y. Takao, K. Kakimoto, New solution method for homogenization analysis and its application to the prediction of macroscopic elastic constants of materials with periodic microstructures, Computers \& Structures, 84 (2006) 991-1001.

[20] E. Andrews, G. Gioux, P. Onck, L. Gibson, Size effects in ductile cellular solids. Part II: experimental results, International Journal of Mechanical Sciences, 43 (2001) 701-713.

[21] C. Foo, G. Chai, L. Seah, Mechanical properties of Nomex material and Nomex honeycomb structure, Composite Structures, 80 (2007) 588-594.

[22] D. Mohr, Mechanism-based multi-surface plasticity model for ideal truss lattice materials, International Journal of Solids and Structures, 42 (2005) 3235-3260.

[23] S. Demiray, W. Becker, J. Hohe, Numerical determination of initial and subsequent yield surfaces of opencelled model foams, International Journal of Solids and Structures, 44 (2007) 2093-2108.

[24] M. Doyoyo, J. Wan Hu, Plastic failure analysis of an auxetic foam or inverted strut lattice under longitudinal and shear loads, Journal of the Mechanics and Physics of Solids, 54 (2006) 1479-1492.

[25] A. Wang, D. McDowell, Yield surfaces of various periodic metal honeycombs at intermediate relative density, International Journal of Plasticity, 21 (2005) 285-320.

[26] R. Hutchinson, N. Fleck, The structural performance of the periodic truss, Journal of the Mechanics and Physics of Solids, 54 (2006) 756-782.

[27] M.S.A. Elsayed, D. Pasini, Analysis of the elastostatic specific stiffness of 2D stretching-dominated lattice materials, Mechanics of Materials, 42 (2010) 709-725.

[28] M.S.A. Elsayed, Multiscale mechanics and structural design of periodic cellular materials, in, MCGILL UNIVERSITY, 2012.

[29] A. Vigliotti, D. Pasini, Linear multiscale analysis and finite element validation of stretching and bending dominated lattice materials, Mechanics of Materials, 46 (2012) 57-68.

[30] A. Vigliotti, D. Pasini, Stiffness and strength of tridimensional periodic lattices, Computer methods in applied mechanics and engineering, 229-232 (2012) 27-43.

[31] A.C. Eringen, Linear theory of micropolar elasticity, Journal of Mathematics and Mechanics, 15 (1966) 909923.

[32] R.S. Lakes, Strongly Cosserat elastic lattice and foam materials for enhanced toughness, Cellular Polymers, 12 (1993) 17.

[33] C. Florence, K. Sab, A rigorous homogenization method for the determination of the overall ultimate strength of periodic discrete media and an application to general hexagonal lattices of beams, European Journal of Mechanics-A/Solids, 25 (2006) 72-97.

[34] F. Pradel, K. Sab, Cosserat modelling of elastic periodic lattice structures, Comptes Rendus de l'Académie des Sciences-Series IIB-Mechanics-Physics-Astronomy, 326 (1998) 699-704.

[35] F. Dos Reis, J. Ganghoffer, Discrete homogenization of architectured materials: Implementation of the method in a simulation tool for the systematic prediction of their effective elastic properties, Technische Mechanik, 30 (2010) 85-109.

[36] M. Assidi, F. Dos Reis, J.-F. Ganghoffer, Equivalent mechanical properties of biological membranes from lattice homogenization, Journal of the Mechanical Behavior of Biomedical Materials, 4 (2011) 1833-1845.

[37] F. Dos Reis, J. Ganghoffer, Equivalent mechanical properties of auxetic lattices from discrete homogenization, Computational Materials Science, 51 (2012) 314-321.

[38] A.L. Kalamkarov, I.V. Andrianov, V.V. Danishevs'kyy, Asymptotic homogenization of composite materials and structures, Applied Mechanics Reviews, 62 (2009) 030802.

[39] B. Hassani, E. Hinton, A review of homogenization and topology optimization III--topology optimization using optimality criteria, Computers \& Structures, 69 (1998) 739-756.

[40] O. Sigmund, Materials with prescribed constitutive parameters: an inverse homogenization problem, International Journal of Solids and Structures, 31 (1994) 2313-2329.

[41] M. Bendsøe, Optimal shape design as a material distribution problem, Structural and Multidisciplinary Optimization, 1 (1989) 193-202. 
[42] N. Kikuchi, M. bendsoe, Generating optimal topologies in structural design using a homogenization method, Computer methods in applied mechanics and engineering, 71 (1988) 197-224.

[43] C.Y. Lin, N. Kikuchi, S.J. Hollister, A novel method for biomaterial scaffold internal architecture design to match bone elastic properties with desired porosity, Journal of Biomechanics, 37 (2004) 623-636.

[44] S. Sturm, S. Zhou, Y.-W. Mai, Q. Li, On stiffness of scaffolds for bone tissue engineering - a numerical study, Journal of Biomechanics, 43 (2010) 1738-1744.

[45] S.J. Hollister, Porous scaffold design for tissue engineering, Nature materials, 4 (2005) 518-524.

[46] N. Takano, Y. Ohnishi, M. Zako, K. Nishiyabu, Microstructure-based deep-drawing simulation of knitted fabric reinforced thermoplastics by homogenization theory, International Journal of Solids and Structures, 38 (2001) 6333-6356.

[47] N. Takano, M. Zako, F. Kubo, K. Kimura, Microstructure-based stress analysis and evaluation for porous ceramics by homogenization method with digital image-based modeling, International Journal of Solids and Structures, 40 (2003) 1225-1242.

[48] R. Guinovart-Díaz, R. Rodríguez-Ramos, J. Bravo-Castillero, F.J. Sabina, R. Dario Santiago, R. Martinez Rosado, Asymptotic analysis of linear thermoelastic properties of fiber composites, Journal of thermoplastic composite materials, 20 (2007) 389-410.

[49] R. Guinovart-Diaz, J. Bravo-Castillero, R. Rodriguez-Ramos, R. Martinez-Rosado, F. Serrania, M. Navarrete, Modeling of elastic transversely isotropic composite using the asymptotic homogenization method. Some comparisons with other models, Materials Letters, 56 (2002) 889-894.

[50] X. Peng, J. Cao, A dual homogenization and finite element approach for material characterization of textile composites, Composites Part B: Engineering, 33 (2002) 45-56.

[51] A. Visrolia, M. Meo, Multiscale damage modelling of 3D weave composite by asymptotic homogenisation, Composite Structures, 95 (2013) 105-113.

[52] S. Jansson, Homogenized nonlinear constitutive properties and local stress concentrations for composites with periodic internal structure, International Journal of Solids and Structures, 29 (1992) 2181-2200.

[53] K. Matsui, K. Terada, K. Yuge, Two-scale finite element analysis of heterogeneous solids with periodic microstructures, Computers \& Structures, 82 (2004) 593-606.

[54] S.A. Khanoki, D. Pasini, Multiscale Design and Multiobjective Optimization of Orthopedic Hip Implants with Functionally Graded Cellular Material, Journal of Biomechanical Engineering, 134 (2012) 031004. [55] A.M. Hayes, A. Wang, B.M. Dempsey, D.L. McDowell, Mechanics of linear cellular alloys, Mechanics of Materials, 36 (2004) 691-713.

[56] R.S. Kumar, D.L. McDowell, Multifunctional design of two-dimensional cellular materials with tailored mesostructure, International Journal of Solids and Structures, 46 (2009) 2871-2885.

[57] A. Evans, J. Hutchinson, N. Fleck, M. Ashby, H. Wadley, The topological design of multifunctional cellular metals, Progress in Materials Science, 46 (2001) 309-328.

[58] L. Liu, J. Yan, G. Cheng, Optimum structure with homogeneous optimum truss-like material, Computers \& Structures, 86 (2008) 1417-1425.

[59] S. Hollister, N. Kikuchi, A comparison of homogenization and standard mechanics analyses for periodic porous composites, Computational Mechanics, 10 (1992) 73-95.

[60] Z. Fang, C. Yan, W. Sun, A. Shokoufandeh, W. Regli, Homogenization of heterogeneous tissue scaffold: A comparison of mechanics, asymptotic homogenization, and finite element approach, Applied Bionics and Biomechanics, 2 (2005) 17-29.

[61] B. Hassani, A direct method to derive the boundary conditions of the homogenization equation for symmetric cells, Communications in numerical methods in engineering, 12 (1996) 185-196.

[62] E. Masoumi Khalil Abad, S. Arabnejad Khanoki, D. Pasini, Fatigue design of lattice materials via computational mechanics: Application to lattices with smooth transitions in cell geometry, International Journal of Fatigue, 47 (2013) 126-136.

[63] N. Takano, M. Zako, T. Okazaki, Efficient modeling of microscopic heterogeneity and local crack in composite materials by finite element mesh superposition method, JSME International Journal Series A, 44 (2001) 602-609. 
[64] N. Takano, M. Zako, Y. Okuno, Multi-scale finite element analysis of porous materials and components by asymptotic homogenization theory and enhanced mesh superposition method, Modelling and Simulation in Materials Science and Engineering, 11 (2003) 137-156.

[65] N. Takano, Y. Okuno, Three-scale finite element analysis of heterogeneous media by asymptotic homogenization and mesh superposition methods, International Journal of Solids and Structures, 41 (2004) 41214135.

[66] S. Ghosh, K. Lee, P. Raghavan, A multi-level computational model for multi-scale damage analysis in composite and porous materials, International Journal of Solids and Structures, 38 (2001) 2335-2385.

[67] R.G. Hutchinson, Mechanics of lattice materials, in: Department of Engineering, University of Cambridge, 2004. 


\section{Table Caption}

Table 1. Yield surfaces as a function of relative density for square, hexagonal, and Kagome unit cells

Table 2. The expressions of the effective mechanical properties obtained in literature for various cell topologies 


\section{Figure Caption}

Figure 1. Cell topologies with the associated RVE considered in this study

Figure 2. Homogenization concept of a cellular structure

Figure 3. Periodic boundary conditions on the opposite edges of the RVE

Figure 4. Effective elastic constants as a function of relative density for the cell topologies: (a) square, (b) hexagonal, (c) Kagome, (d) triangle, (e) mixed square/triangular A, and (f) mixed square/triangular B Figure 5. Yield strength as a function of relative density for: (a) square, (b) hexagonal, (c) Kagome, (d) triangle, (e) mixed square/triangular A, and (f) mixed square/triangular B cells

Figure 6. Yield surface of an hexagonal cell honeycomb under combined in-plane stress state $\left(\sigma_{x}, \sigma_{y}\right.$ and $\tau_{x y}$ ) for a relative density $\rho=50 \%$

Figure 7. Yield surface of a square cell honeycomb under combined in-plane stress state $\left(\sigma_{x}, \sigma_{y}\right.$ and $\tau_{x y}$ ) for a relative density $\rho=50 \%$

Figure 8. Yield surface of a Kagome cell honeycomb under combined in-plane stress state $\left(\sigma_{x}, \sigma_{y}\right.$ and $\left.\tau_{x y}\right)$ for a relative density $\rho=30 \%$

Figure 9. Effective elastic constants for (a) square, (b) hexagonal, (c) Kagome, (d) triangle, (e) mixed square/triangular $\mathrm{A}$, and (f) mixed square/triangular $\mathrm{B}$, for relative density below $0.3(\rho<0.3)$. The closed-form expressions of the effective elastic constants obtained by Gibson and Ashby [3], Wang and McDowell [6], Elsayed [28], and Vigliotti and Pasini, [29] are plotted.

Figure 10. Relative error between the effective elastic constants obtained via the closed-form expressions given in $[3,6,28,29]$ and those obtained by asymptotic homogenization for different cell topologies, (a) square, (b) hexagonal, (c) Kagome, (d) triangle, (e) mixed square/triangular A, and (f) mixed square/triangular $\mathrm{B}$, for the range of relative density lower than $0.3(\rho<0.3)$. 
Figure 11. Yield strength as a function of relative density lower than $0.3(\rho<0.3)$ for: (a) square, (b) hexagonal, (c) Kagome, (d) triangle, (e) mixed square/triangular A, and (f) mixed square/triangular B. The closed-form expressions plotted in figure are those obtained by Gibson and Ashby [3], Wang and McDowell [6], and Vigliotti and Pasini [29].

Figure 12. Relative error between the initial yield strength obtained via the closed-form expressions given in $[3,6,29]$ and those obtained by asymptotic homogenization for (a) square, (b) hexagonal, (c) Kagome, (d) triangle, (e) mixed square/triangular A, and (f) mixed square/triangular B, at density lower than $0.3(\rho<0.3)$. 


\begin{tabular}{|c|c|c|c|c|}
\hline \multicolumn{2}{|c|}{ a) Bending dominated } & Kagome & Triangular & $\begin{array}{c}\text { Mixed } \\
\text { triangular A }\end{array}$ \\
\hline Square & Hexagonal & Kriangular B \\
\hline
\end{tabular}

Figure 1 Cell topologies with the associated RVE considered in this study 


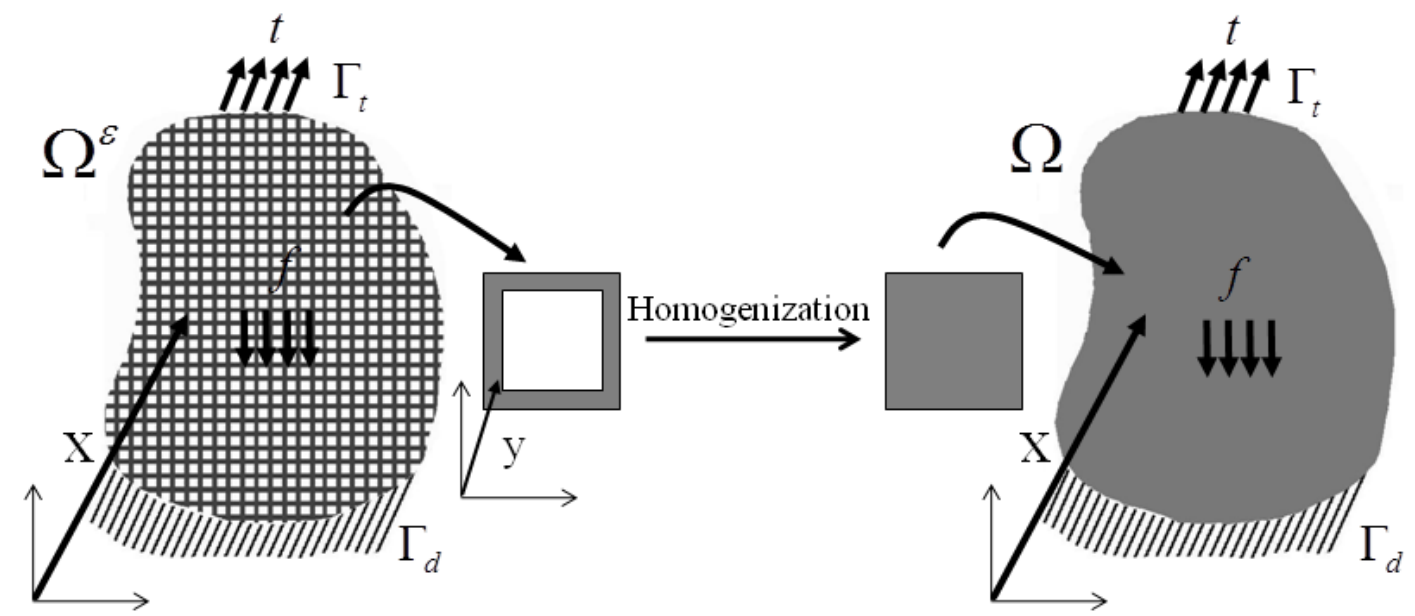

Figure 2: Homogenization concept of a cellular structure 


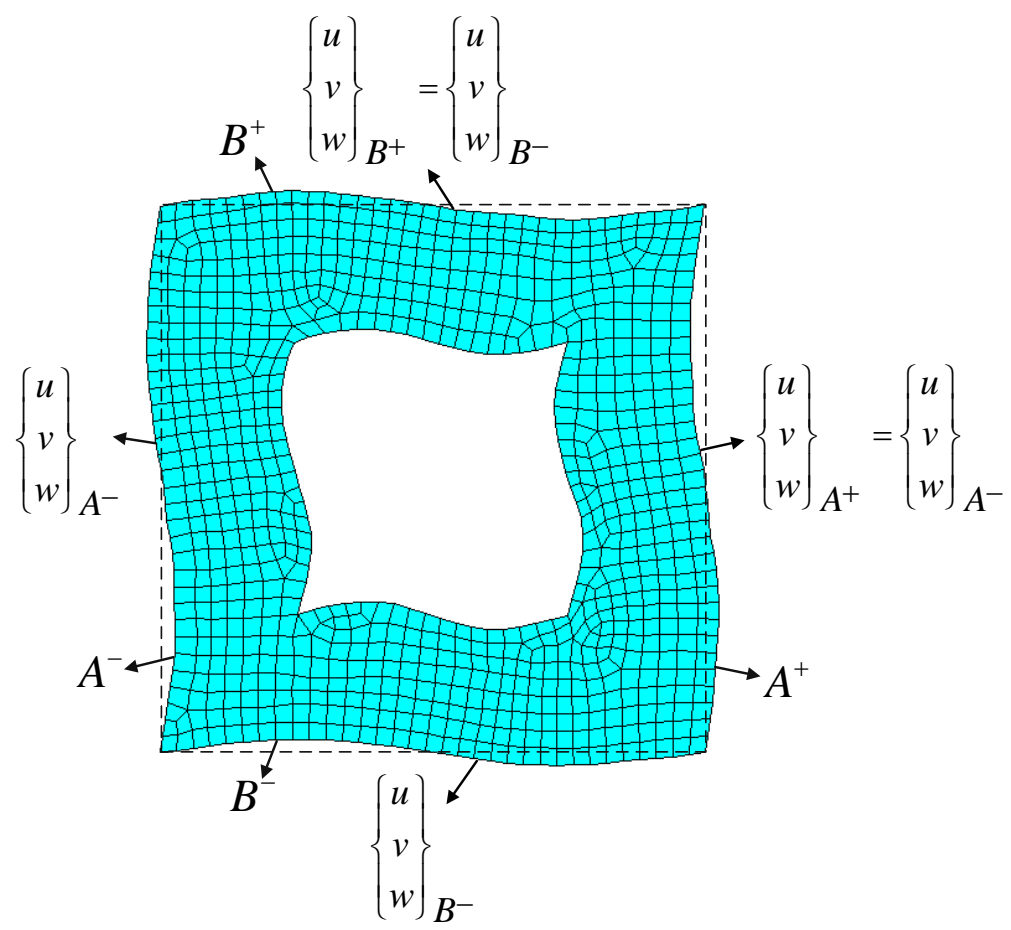

Figure 3: Periodic boundary conditions on the opposite edges of the RVE. 
(a)

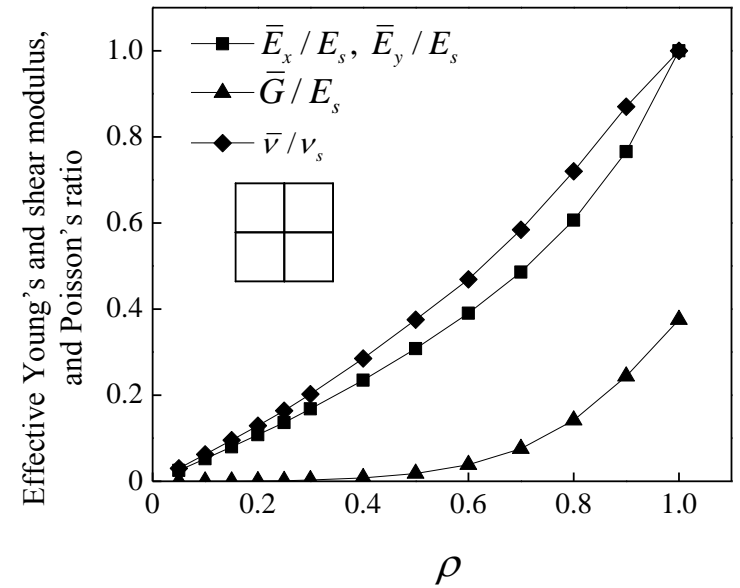

(c)

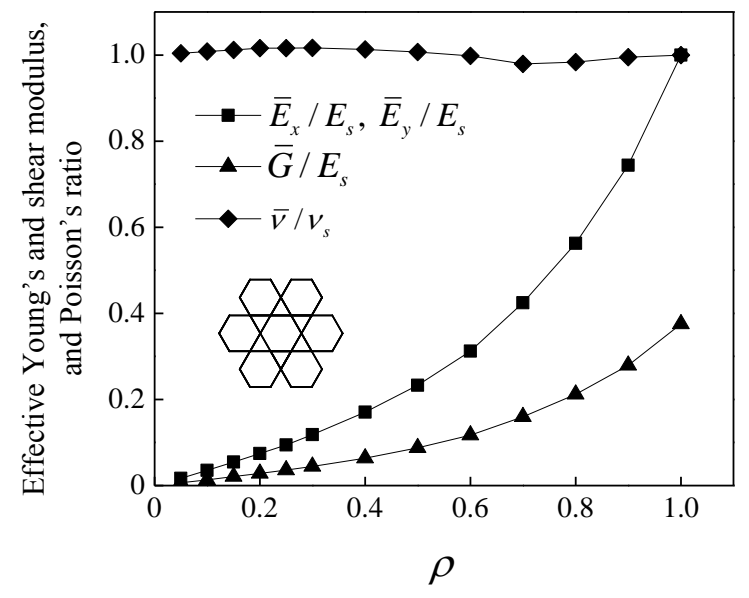

(e)

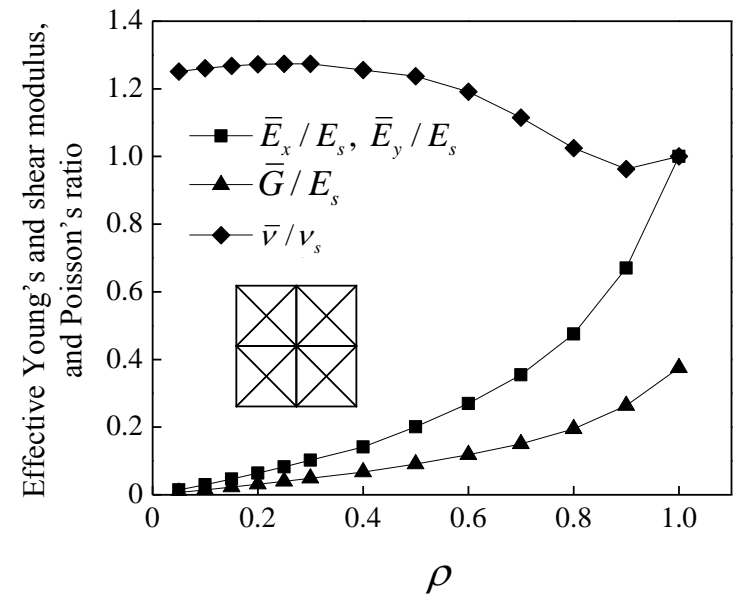

(b)

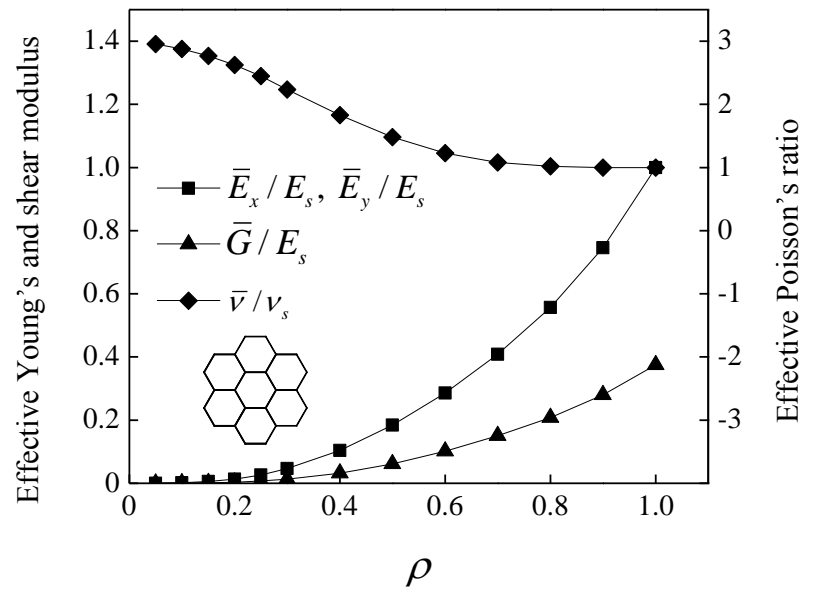

(d)

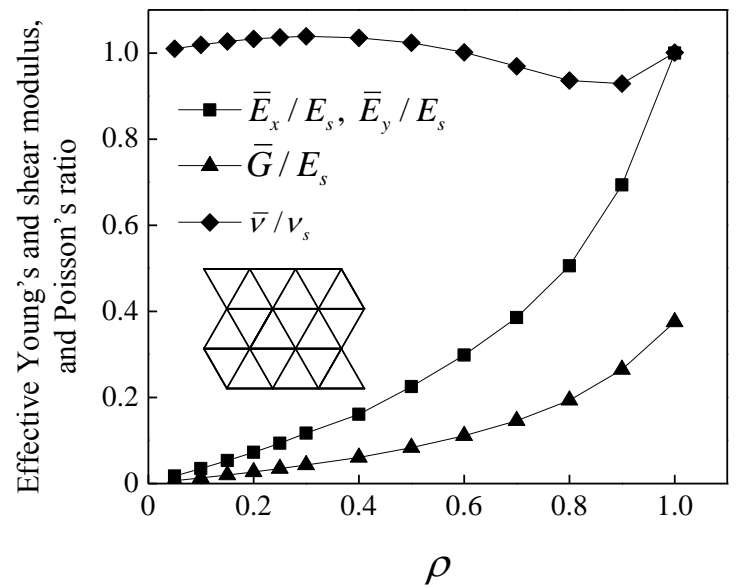

(f)

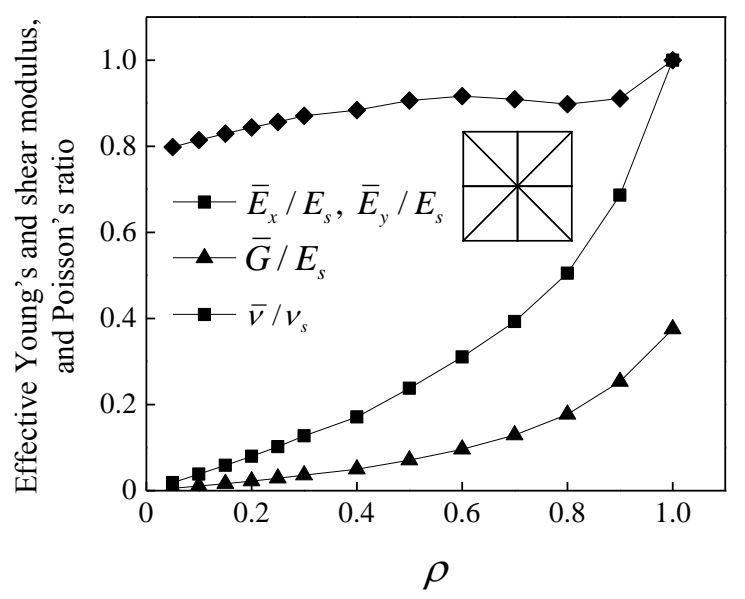

Figure 4: Effective elastic constants as a function of relative density for the cell topologies: (a) square, (b) hexagonal, (c) Kagome, (d) triangle, (e) mixed square/triangular A, and (f) mixed square/triangular B 
(a)

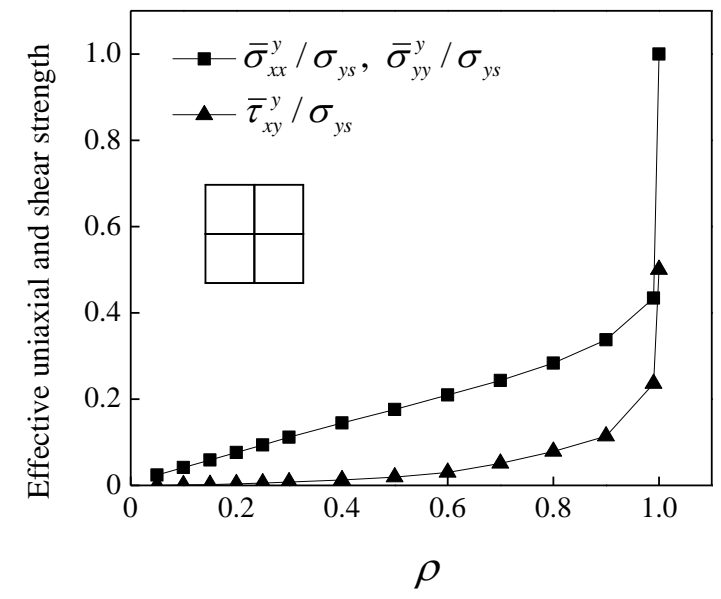

(c)

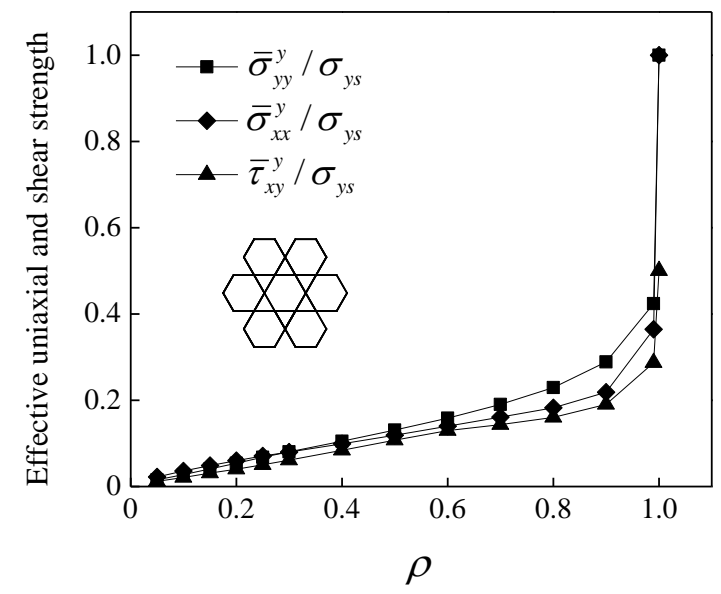

(e)

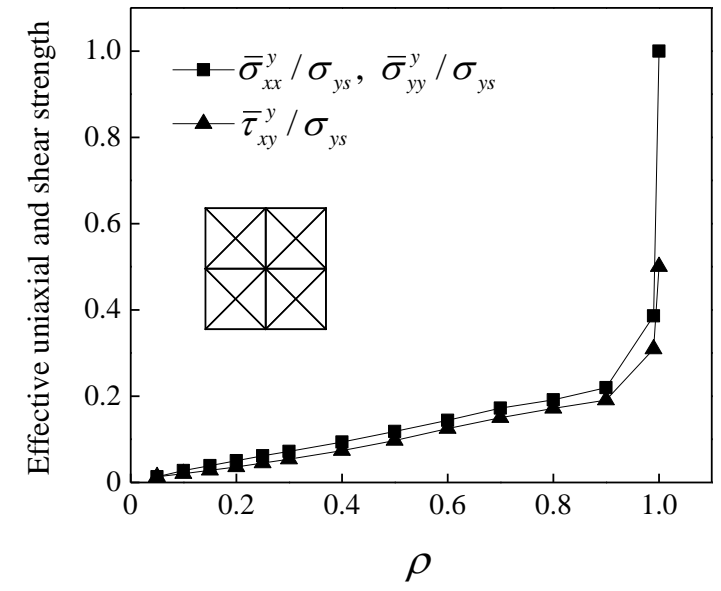

(b)

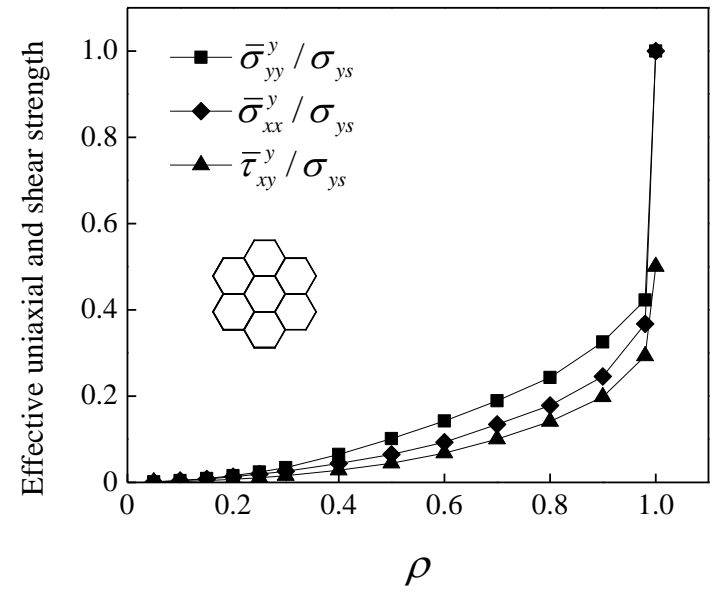

(d)

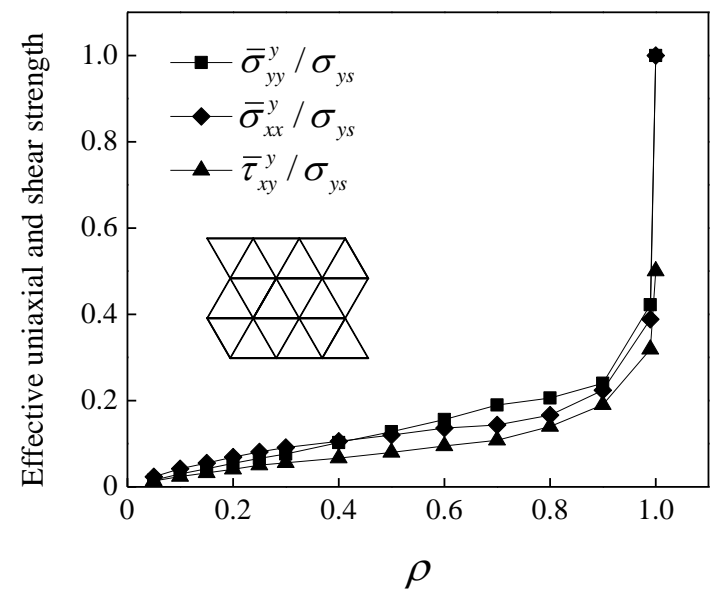

(f)

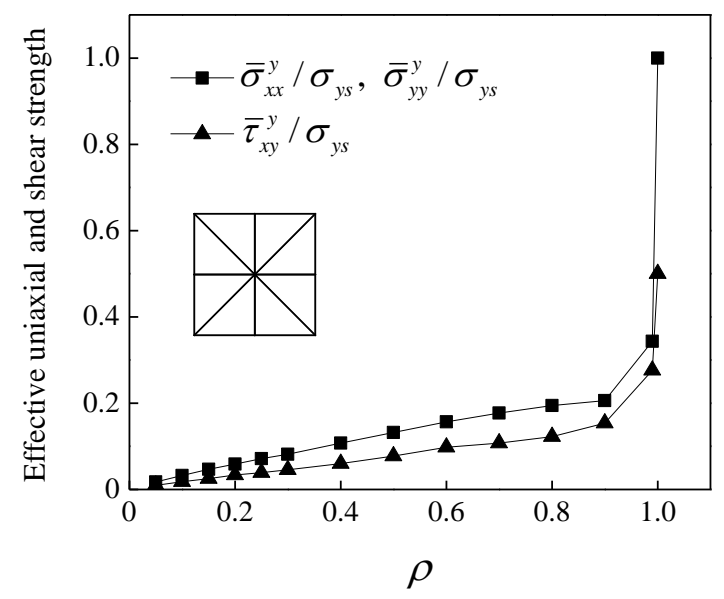

Figure 5: Yield strength as a function of relative density for: (a) square, (b) hexagonal, (c) Kagome, (d) triangle, (e) mixed square/triangular A, and (f) mixed square/triangular B cells 

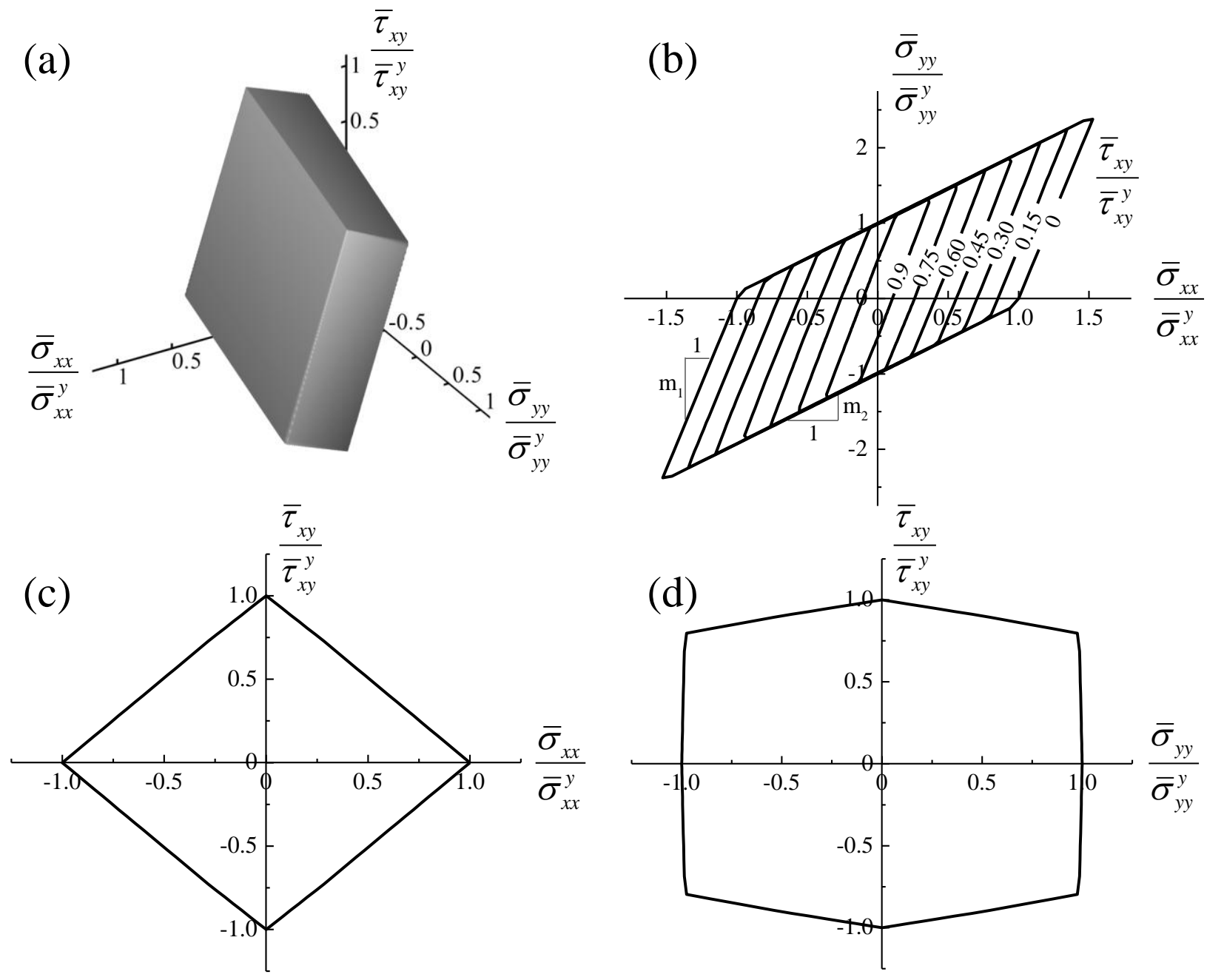

Figure 6: Yield surface of an hexagonal cell honeycomb under combined in-plane stress state $\left(\sigma_{x}, \sigma_{y}\right.$ and $\tau_{x y}$ ) for a relative density $\rho=50 \%$. 

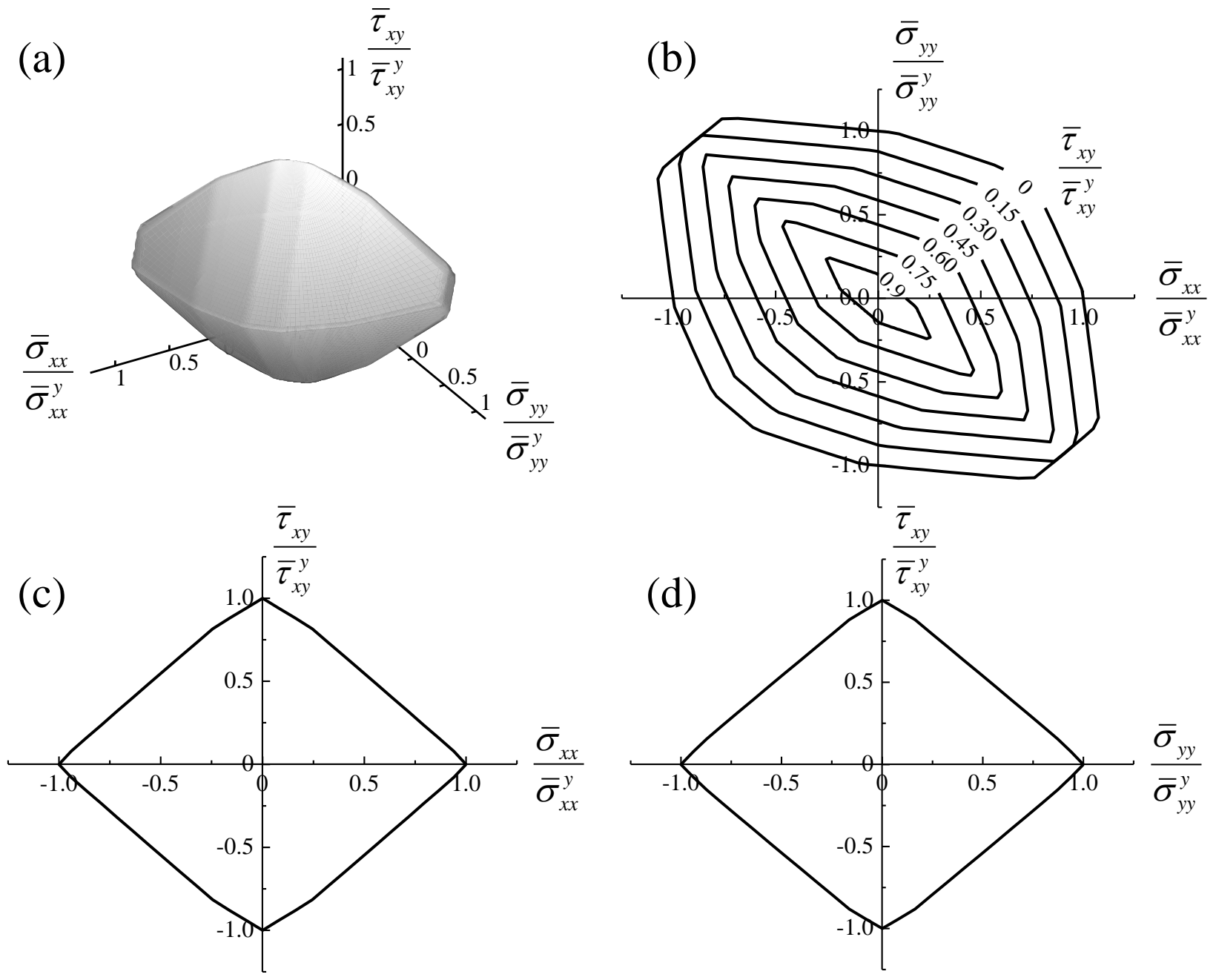

Figure 7: Yield surface of a square cell topology under combined multiaxial macroscopic stress state ( $\bar{\sigma}_{x x}, \bar{\sigma}_{y y}$ and $\left.\bar{\tau}_{x y}\right)$ for a relative density $\rho=50 \%$. 

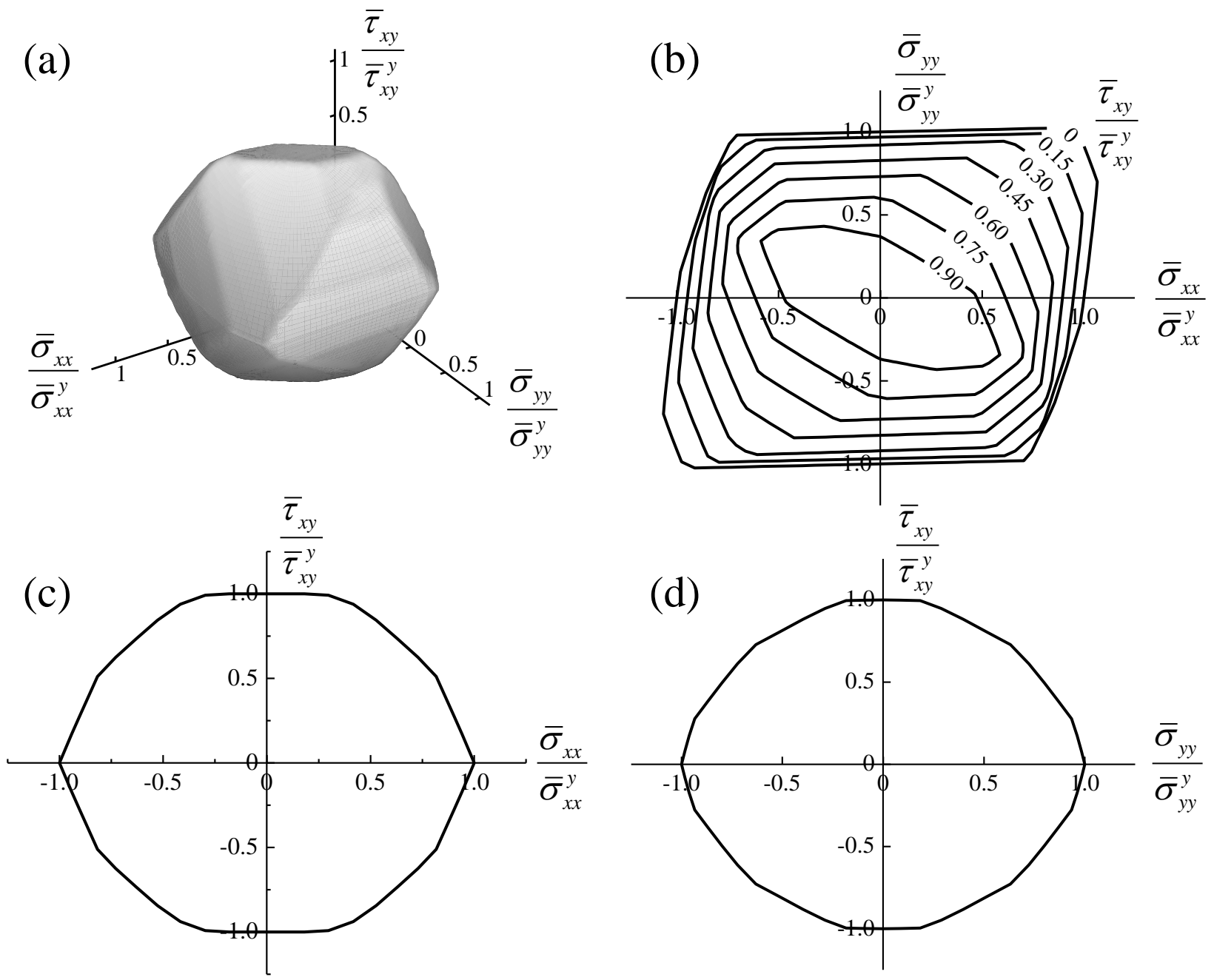

Figure 8: Yield surface of a Kagome cell honeycomb under combined in-plane stress state $\left(\sigma_{x}, \sigma_{y}\right.$ and $\tau_{x y}$ ) for a relative density $\rho=30 \%$. 
(a)

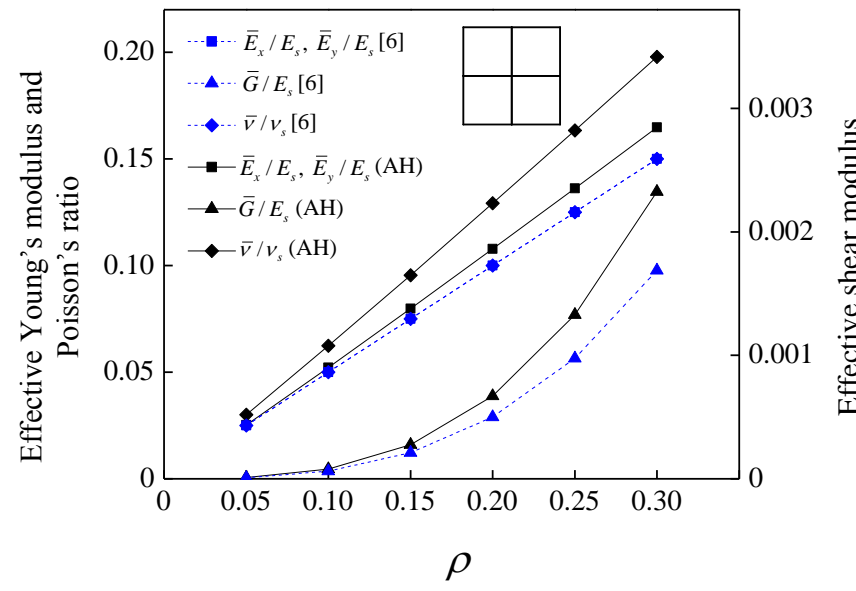

(c)

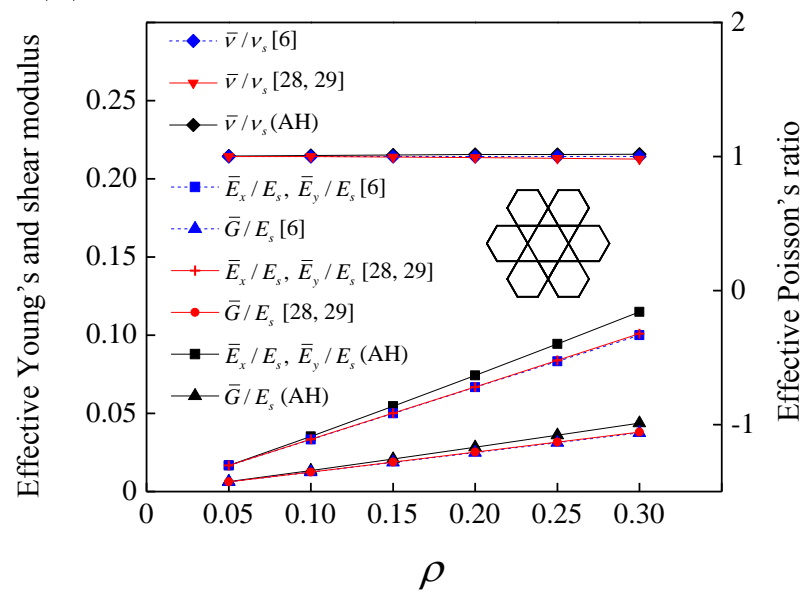

(e)

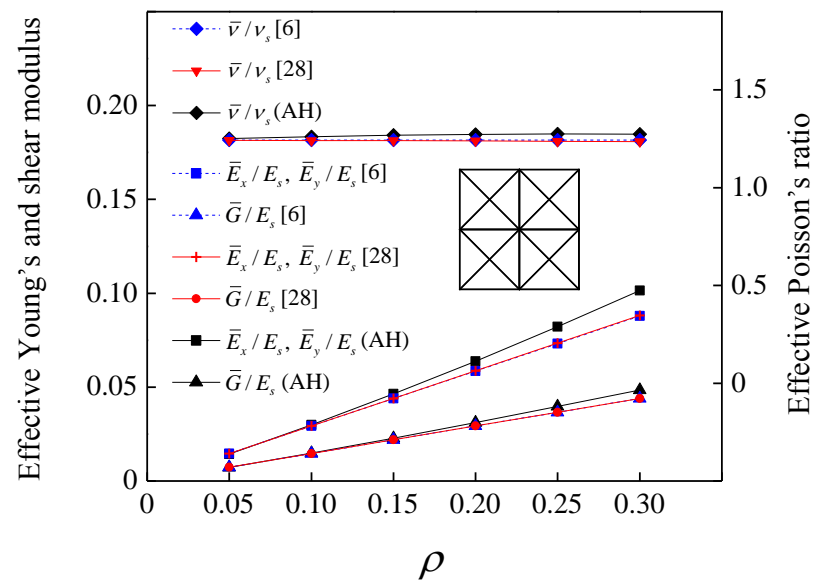

(b)

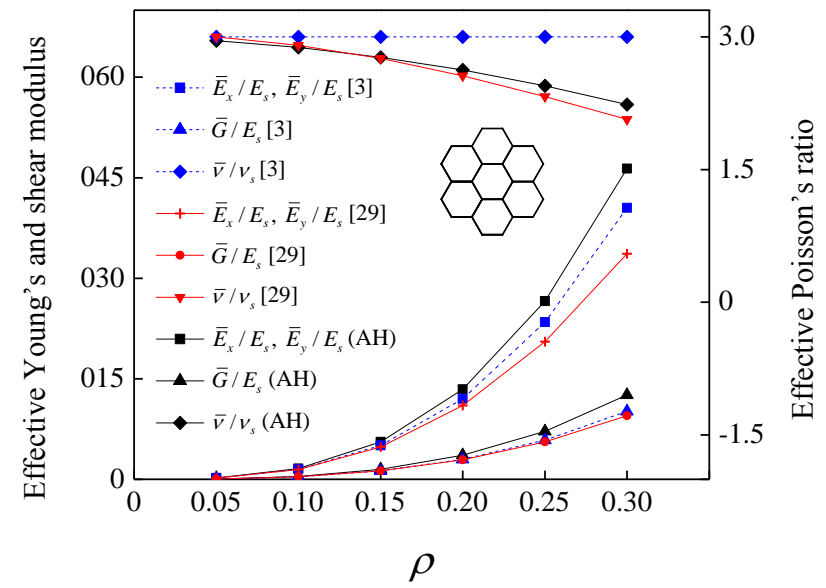

(d)

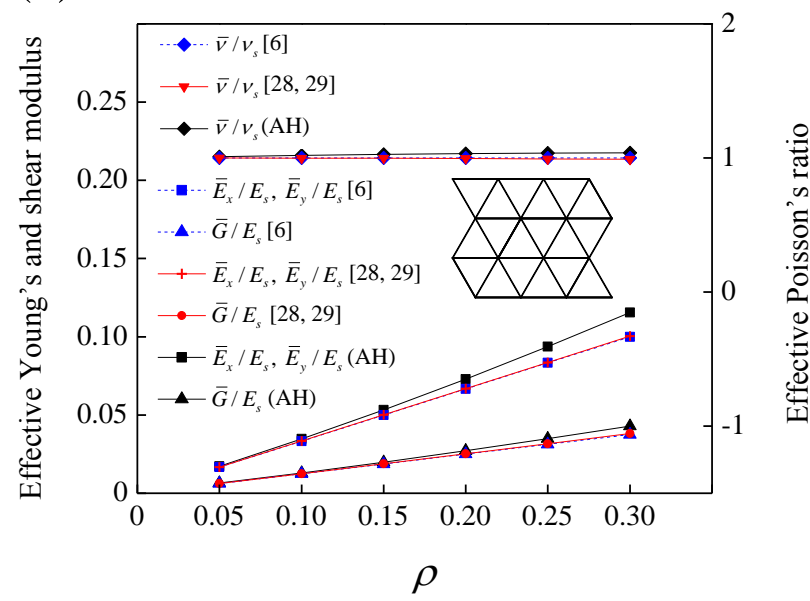

(f)

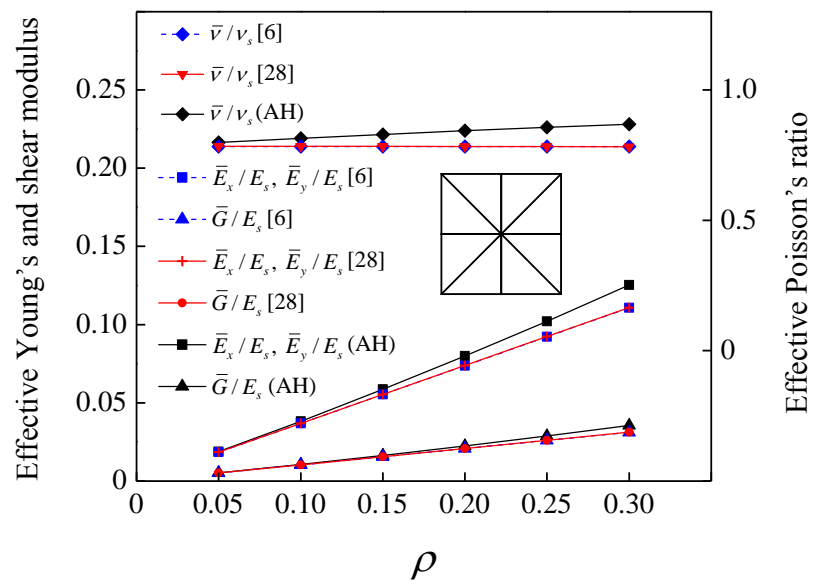

Figure 9: Effective elastic constants for (a) square, (b) hexagonal, (c) Kagome, (d) triangle, (e) mixed square/triangular $\mathrm{A}$, and (f) mixed square/triangular $\mathrm{B}$, for relative density below $0.3(\rho<0.3)$. The closed-form expressions of the effective elastic constants obtained by Gibson and Ashby [3], Wang and McDowell [6], Elsayed [28], and Vigliotti and Pasini [29] are plotted. 
(a)

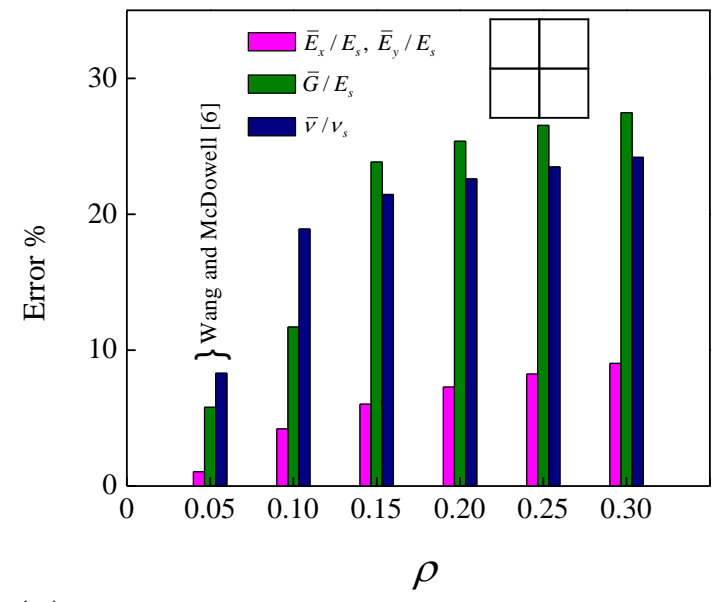

(c)

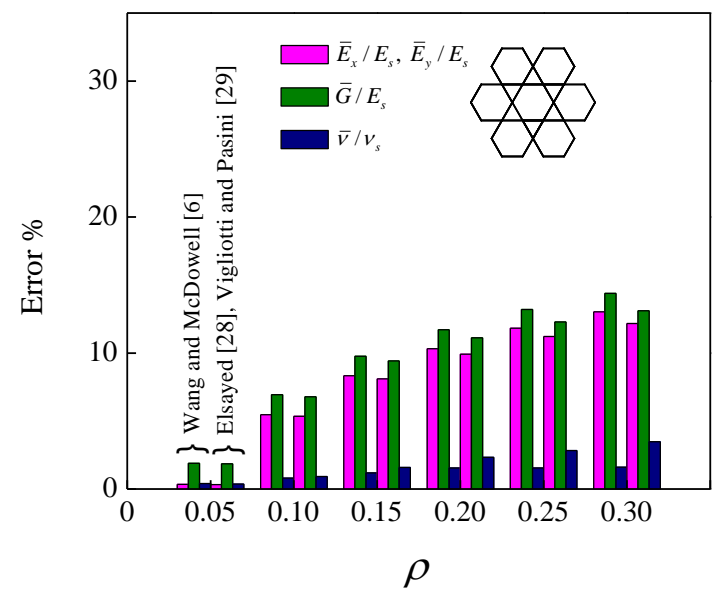

(e)

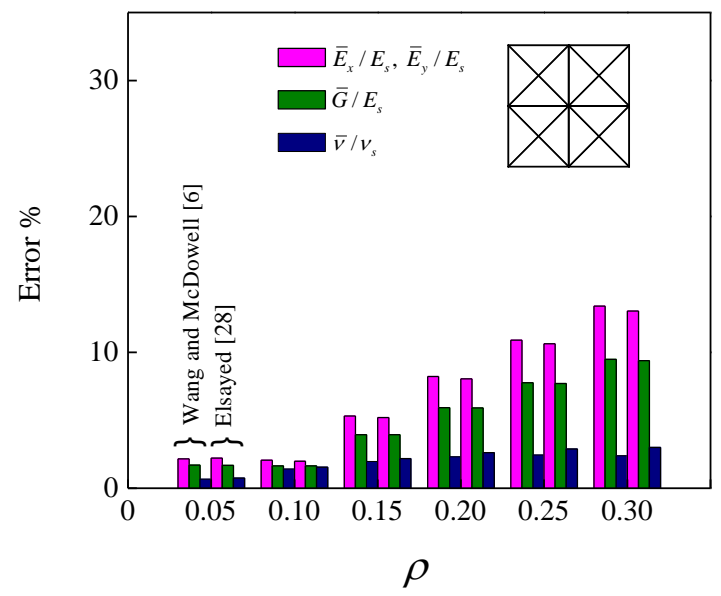

(b)

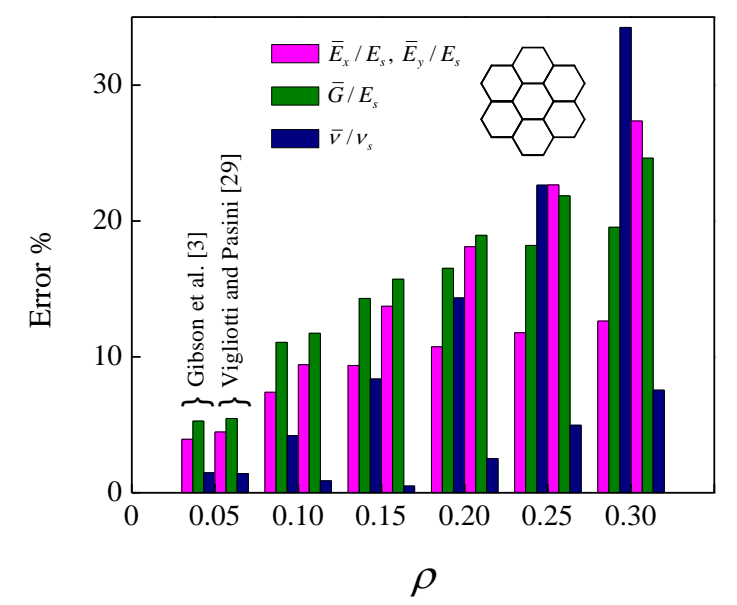

(d)

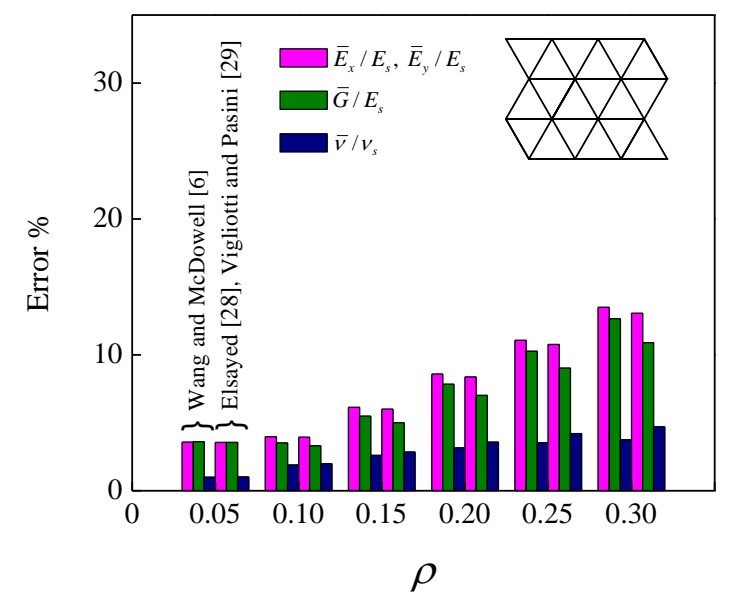

(f)

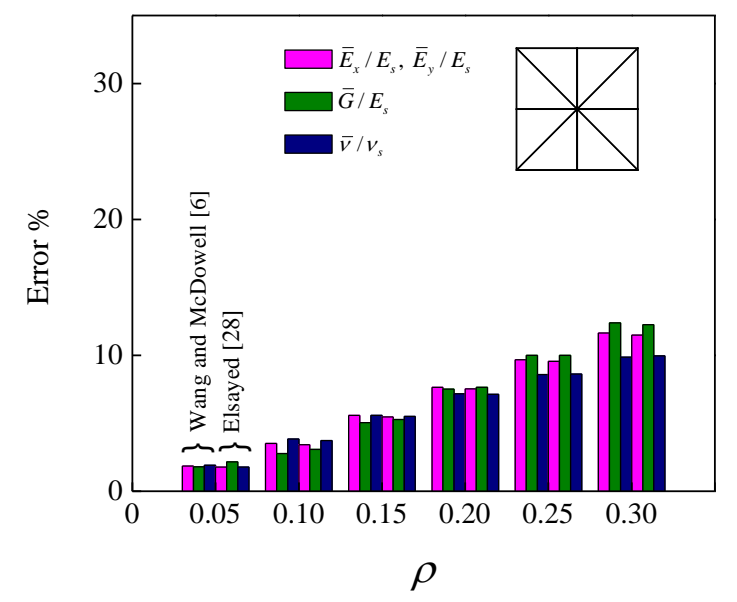

Figure 10: Relative error between the effective elastic constants obtained via the closed-form expressions given in [3, 6, 28, 29] and those obtained by asymptotic homogenization for different cell topologies, (a) square, (b) hexagonal, (c) Kagome, (d) triangle, (e) mixed square/triangular A, and (f) mixed square/triangular $\mathrm{B}$, for the range of relative density lower than $0.3(\rho<0.3)$. 
(a)

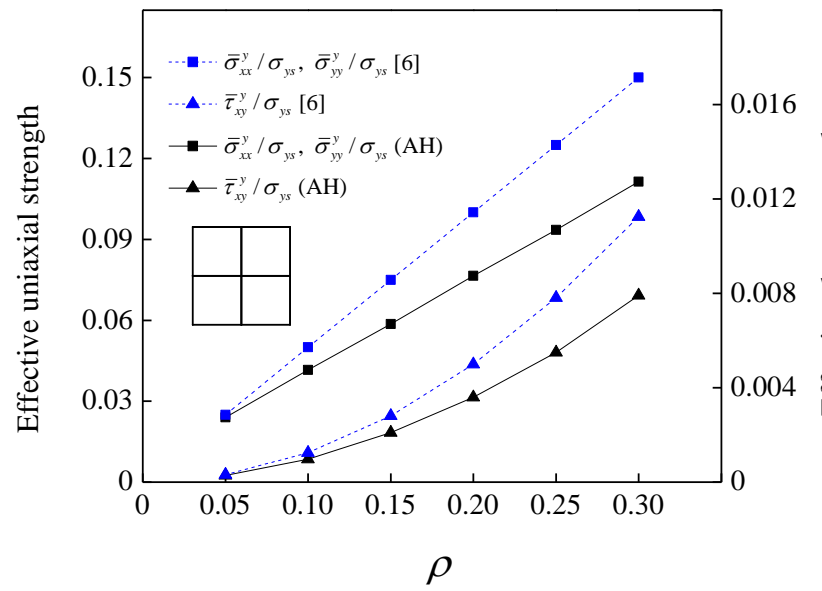

(c)

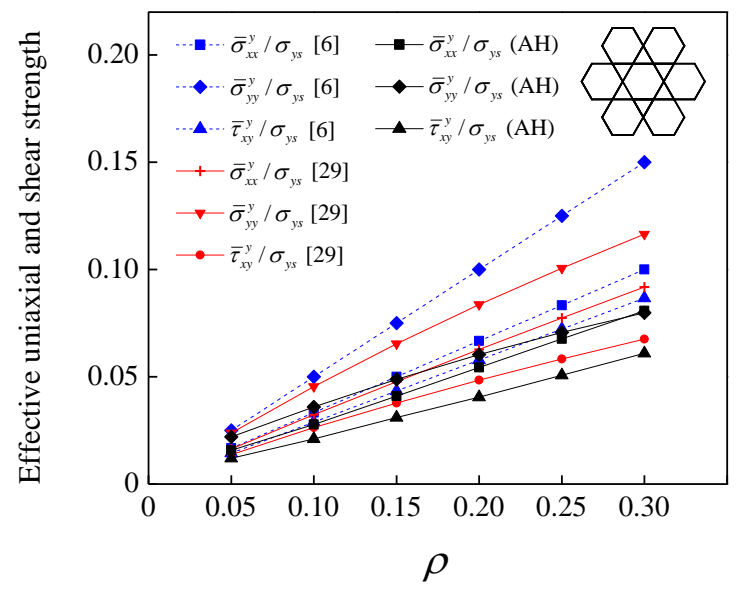

(e)

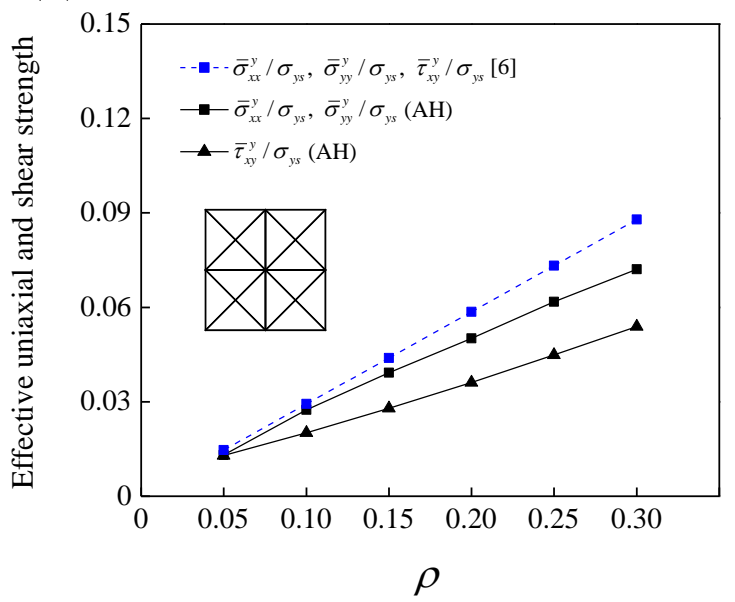

(b)

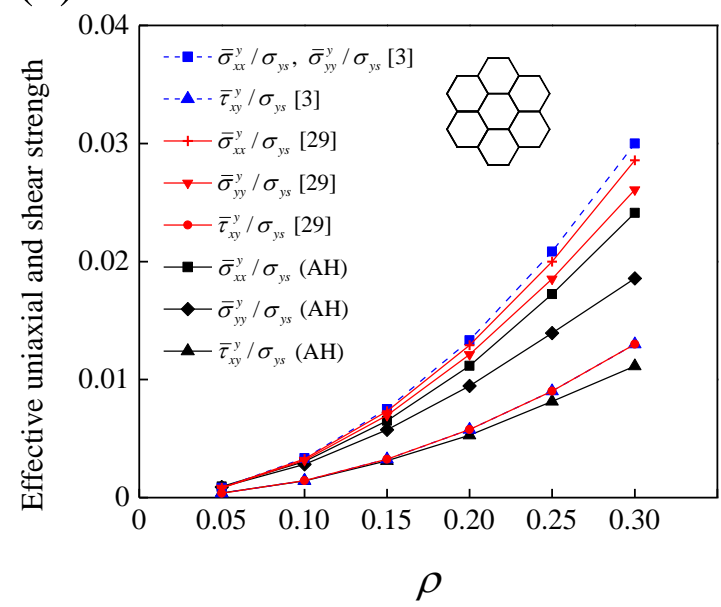

(d)

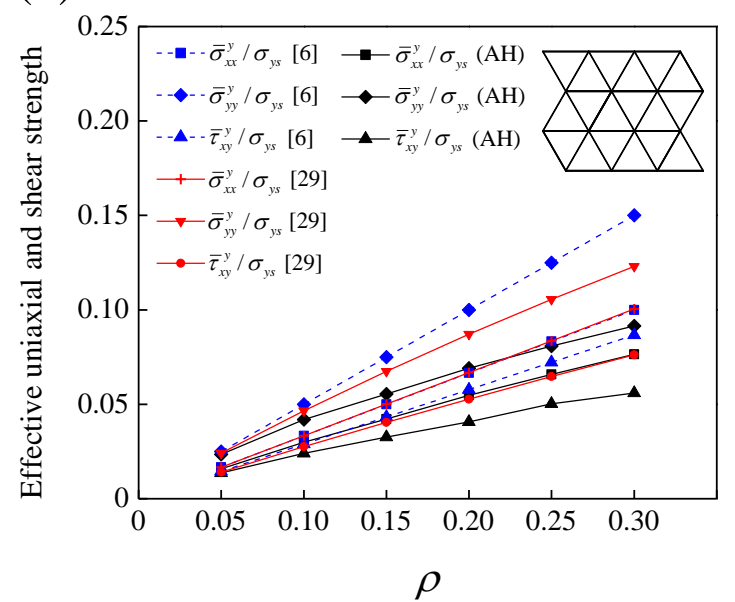

(f)

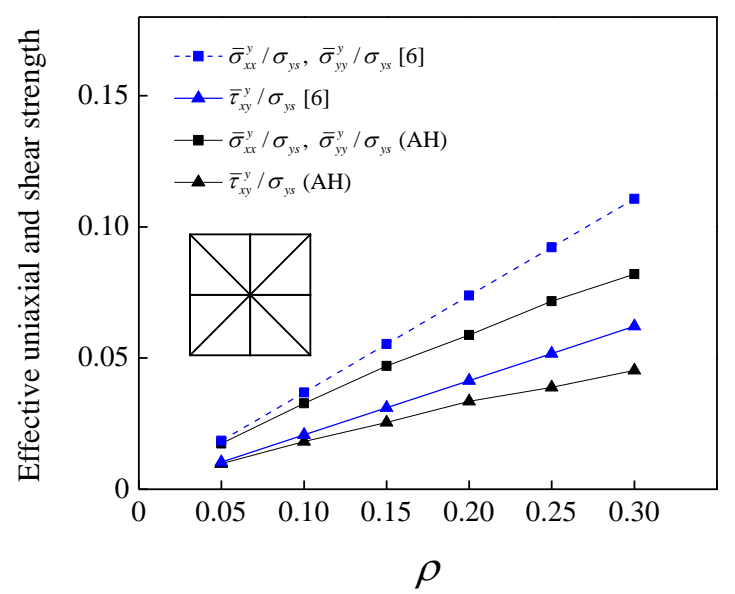

Figure 11: Yield strength as a function of relative density lower than $0.3(\rho<0.3)$ for: (a) square, (b) hexagonal, (c) Kagome, (d) triangle, (e) mixed square/triangular A, and (f) mixed square/triangular B. The closed-form expressions plotted in figure are those obtained by Gibson and Ashby [3], Wang and McDowell [6], and Vigliotti and Pasini [29]. 
(a)

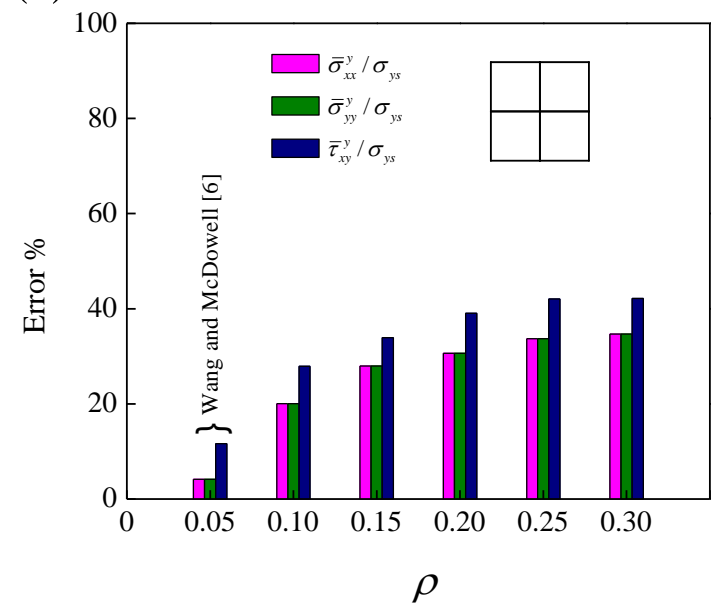

(c)

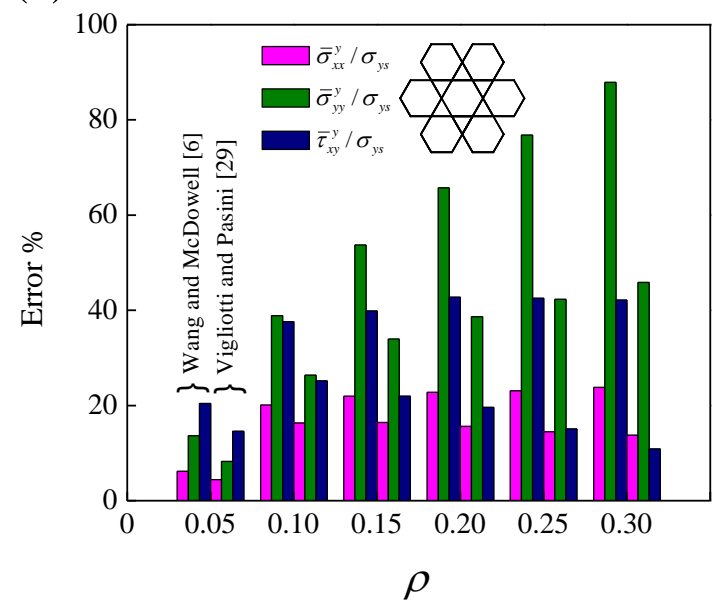

(e)

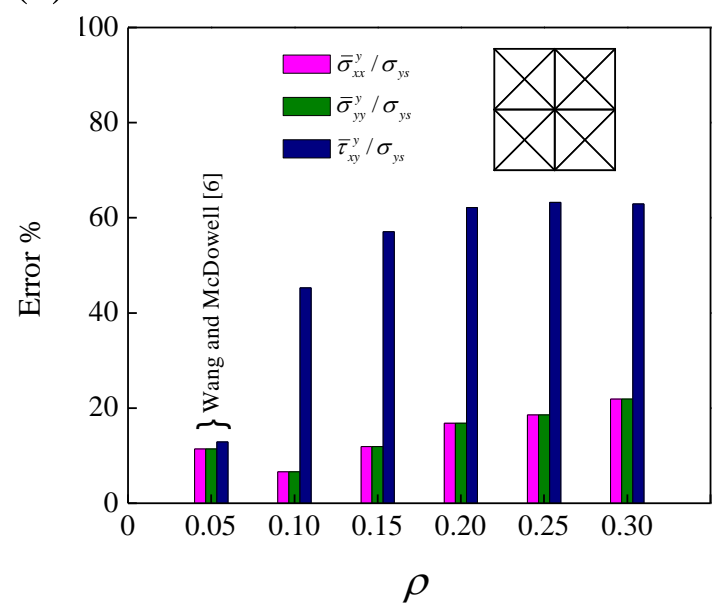

(b)

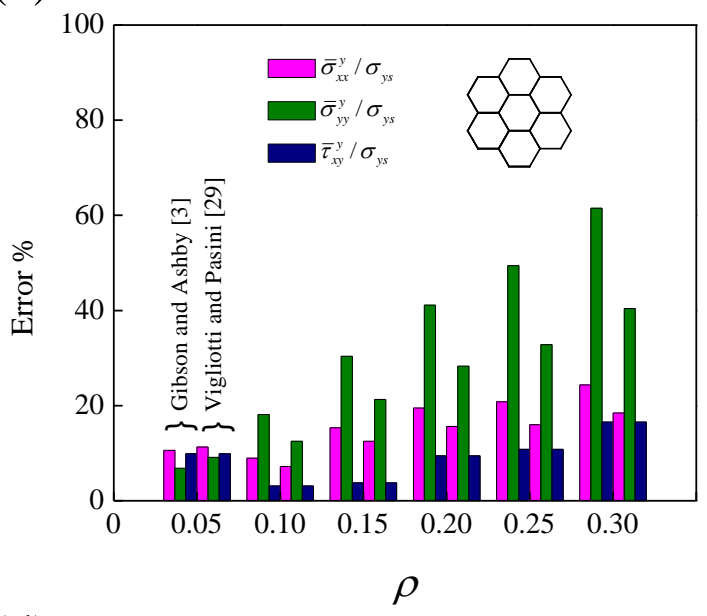

(d)

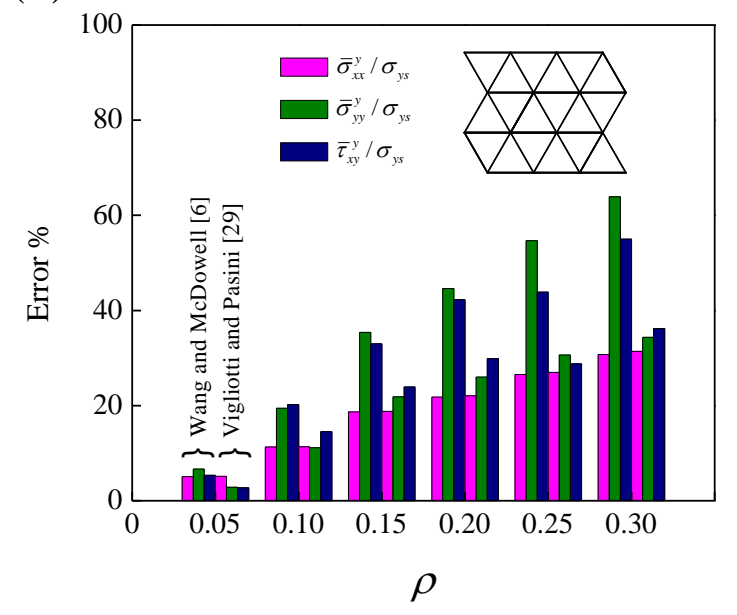

(f)

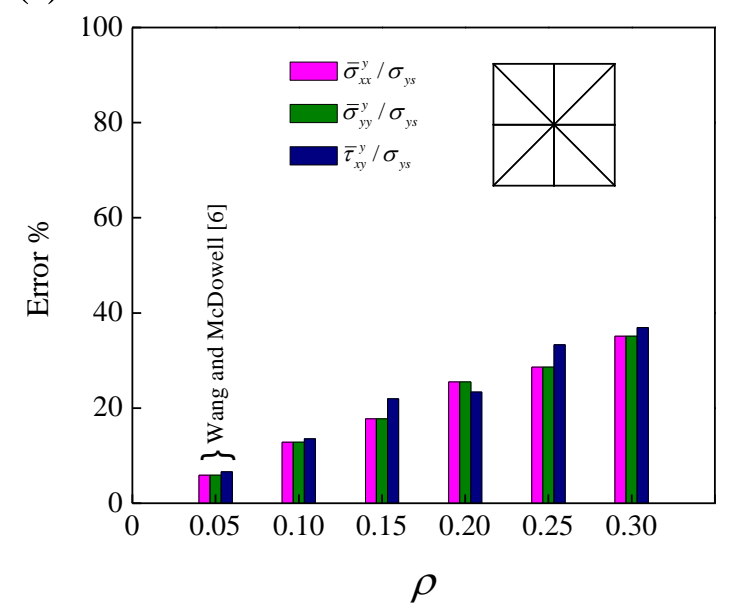

Figure 12: Relative error between the yield strength obtained via the closed-form expressions given in $[3,6,29]$ and those obtained by asymptotic homogenization for (a) square, (b) hexagonal, (c) Kagome, (d) triangle, (e) mixed square/triangular A, and (f) mixed square/triangular B, at density lower than 0.3 ( $\rho<0.3)$. 
Table 1: Yield surfaces as a function of relative density for square, hexagonal, and Kagome unit cells

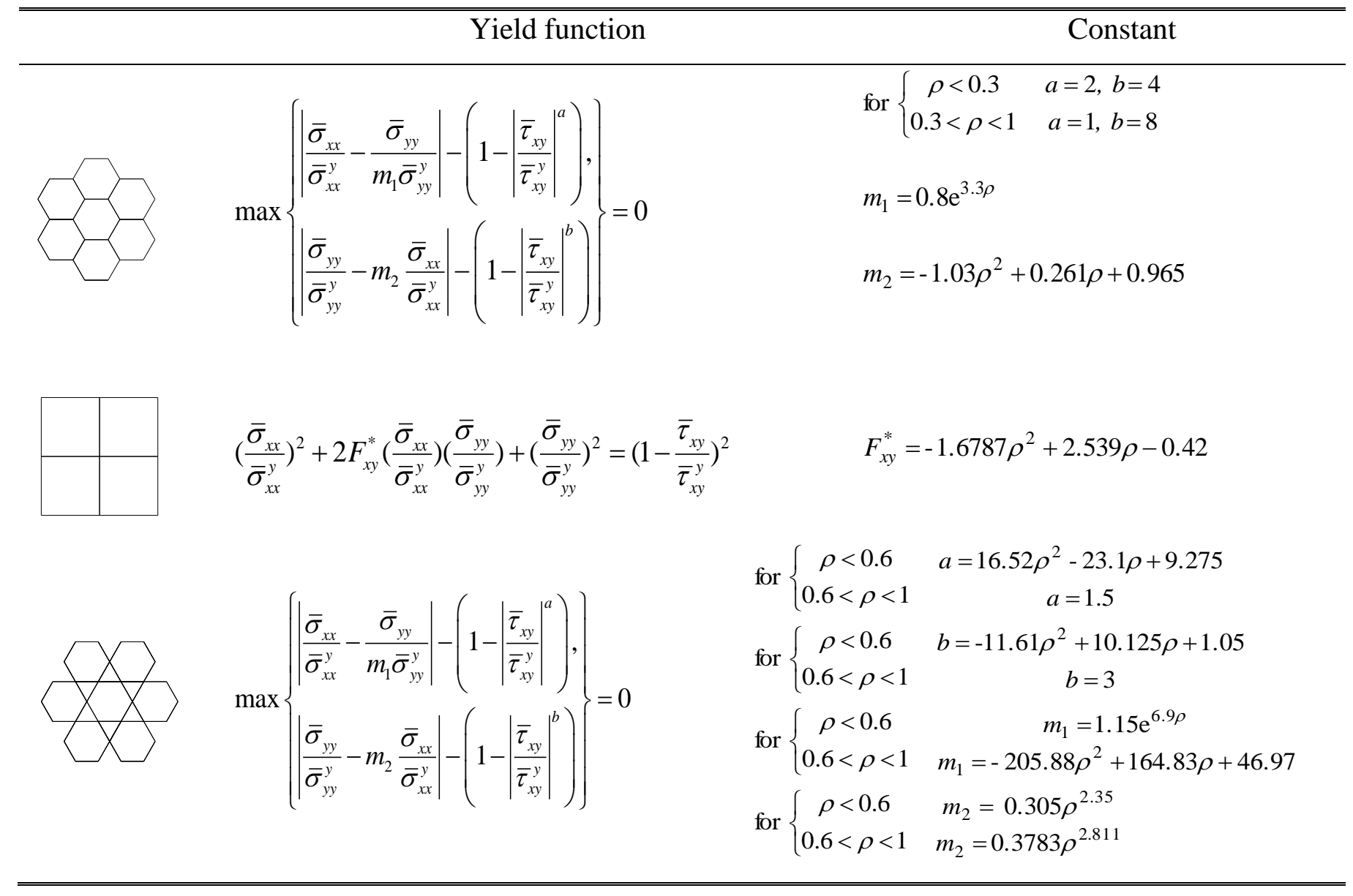


Table 2: The expressions of the effective mechanical properties obtained in literature for various cell topologies

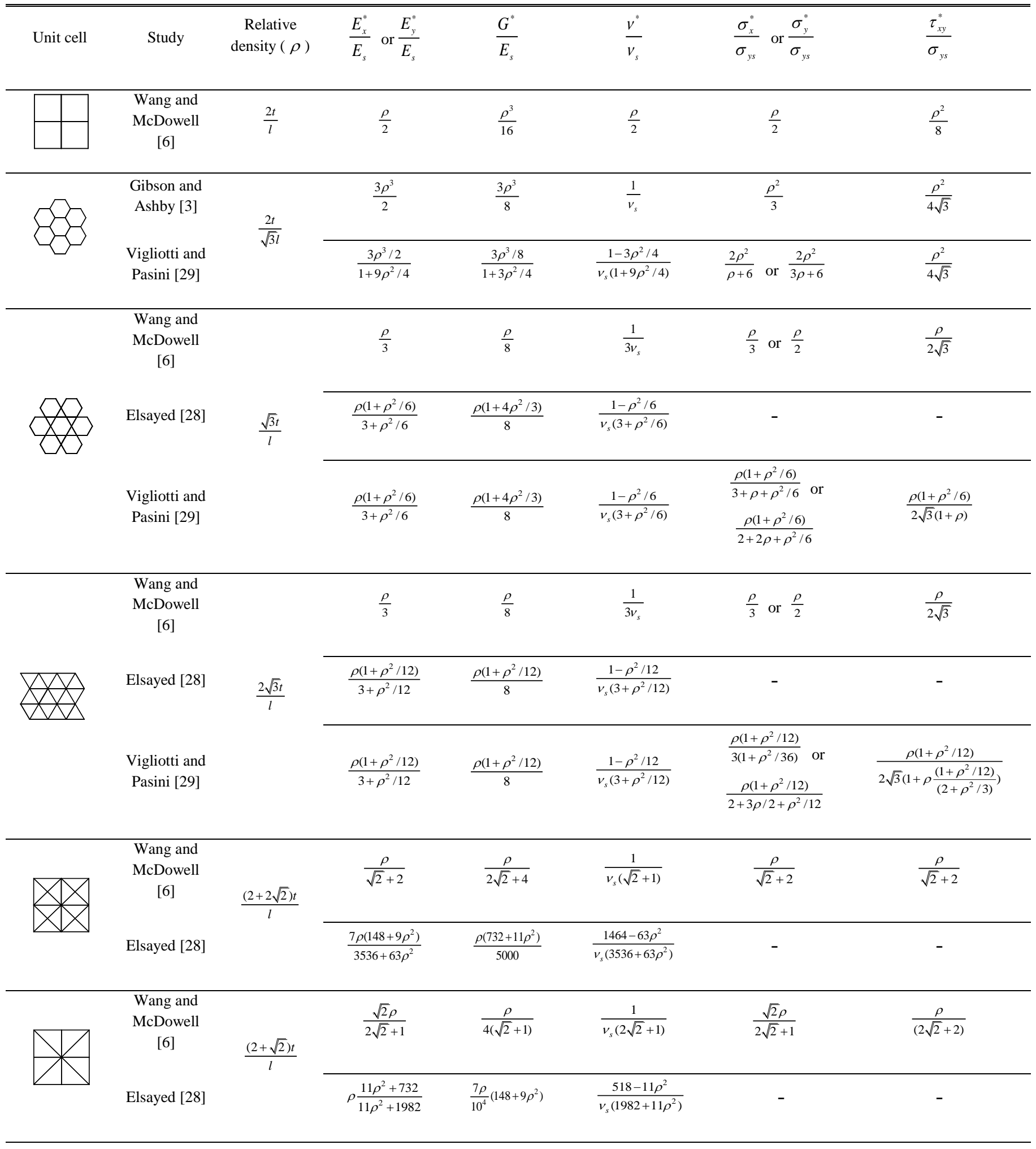

